\title{
Individual function of the ancestral prohormone convertase PC1/3 in Tribolium larval growth and moulting highlights major evolutionary changes between beetle and fly neuroendocrine systems
}

\section{Sonja Fritzsche}

Georg-August-Universitat Gottingen

Vera Sophie Hunnekuhl ( $\sim$ vera.terblanche@uni-goettingen.de )

Georg-August-Universitat Gottingen https://orcid.org/0000-0002-9100-2161

\section{Research}

Keywords: Tribolium castaneum. insect evolution, prohormone convertases, PC1/3, PC2/amontillado, larval development, neuroendocrine system

Posted Date: October 9th, 2020

DOl: https://doi.org/10.21203/rs.3.rs-88522/v1

License: (c) (1) This work is licensed under a Creative Commons Attribution 4.0 International License.

Read Full License 
Individual function of the ancestral prohormone convertase PC1/3 in Tribolium larval growth and moulting highlights major evolutionary changes between beetle and fly neuroendocrine systems

\section{Sonja Fritzsche ${ }^{1} \&$ Vera S. Hunnekuhl*1}

${ }^{1}$ Johann-Friedrich-Blumenbach Institute, GZMB, Göttingen University, Germany

*author for correspondence: Vera Sophie Hunnekuhl; vera.terblanche@uni-goettingen.de co-author: Sonja Fritzsche; sonja.fritzsche@stud.uni-goettingen.de

\section{Abstract}

Background: The insect neuroendocrine system acts in the regulation of physiology, development and growth. Molecular evolution of this system hence has the potential to allow for major biological differences between insect groups. Two prohormone convertases, PC1/3 and PC2, are found in animals and both function in the processing of neuropeptide precursors in the vertebrate neurosecretory pathway. Whereas PC2-function is conserved between the fly Drosophila and vertebrates, ancestral PC1/3 was lost in the fly lineage and has not been functionally studied in any protostome. Results: In order to understand its original functions and the changes accompanying the gene loss in the fly, we investigated PC1/3 and PC2 expression and function in the beetle Tribolium castaneum. We found that PC2 is broadly expressed in the nervous system, whereas surprisingly, PC1/3 expression is restricted to specific cell groups in the posterior brain and suboesophageal ganglion. Both proteases have parallel but non-redundant functions in adult beetles' viability and fertility. Female infertility following RNAi is caused by a failure to deposit sufficient nutritive material to the developing oocytes. Larval RNAi of both genes produced moulting defects where the larvae were not able to shed their old cuticle and became 'locked-in'. Unexpectedly, PC1/3 RNAi larvae went through supernumerary larval moults despite minimal to zero weight gain. Conclusions: Our results indicate a yet unknown molecular mechanism for the coupling of moulting and growth in insect larvae. Conservation of the evolutionary ancient $\mathrm{PC} 1 / 3$ gene in most insect groups 
suggests conserved function and that its loss in dipterans may have been accompanied by major changes in the coordination of larval growth and moulting.

\section{Keywords}

Tribolium castaneum; insect evolution; prohormone convertases; PC1/3; PC2/amontillado; larval development; neuroendocrine system

\section{Background}

A largely conserved gene inventory and similarity of gene function has been observed even between distant animal taxa reflecting a high degree of conservation in genetic control of biology. At the same time, frequent gene loss and duplications are thought to shape divergent animal body plans and niche adaptations. On the other hand, functional divergence of paralogues likewise can drive adaptational macroevolution [1-3]. However, two questions remain poorly studied. First, what are the biological consequences of a drastic change like the loss of a gene acting in a highly conserved process? Second, in how far are the few classic model systems representative for their clade with respect to processes affected by gene loss? Functional genetic work in other representatives of the respective phylum with more conservative genome organisation helps elucidating ancestral gene function and is required for comparisons with more distantly related taxa. In addition, this type of comparative approach reveals the biological changes caused by specific gene loss, or on the factors that made a specific gene dispensable.

Signalling through neuropeptides represents one example of an ancient pathway that is highly conserved between vertebrate and invertebrate taxa [4]. Many aspects of animal life such as life cycle, maturation processes, metabolism and behaviour are orchestrated by the neurosecretory pathway. Nevertheless, this pathway must allow for evolutionary change as well. Due to its wide-ranging regulatory functions, evolutionary modifications that affect the neurosecretory output have the potential to cause major evolutionary changes. Therefore, understanding the molecular evolution of the neurosecretory system is likely to yield insights on major evolutionary events that shaped the biology of an animal lineage.

Neuropeptides secreted from the central nervous system and associated glands are a wellstudied. In many cases orthologues of the hormone encoding genes and their receptors can 
be recognized between invertebrate and vertebrate taxa $[5,6]$. These neuropeptides are commonly transcribed and translated as large precursor molecules (prohormones) that are, before secretion, post-translationally processed by specialized modifying enzymes. Central to the processing of the precursor molecules is internal cleavage at dibasic sites (often RR, KR) by subtilisin-like prohormone convertases of the PC family (see Hoshino and Lindberg 2012; Wegener et al. 2011). Two proteases, $\mathrm{PC} 1 / 3$ and $\mathrm{PC} 2$ are expressed predominantly in the central nervous system and neuroendocrine glands and are specifically involved in the processing of neuropeptides. Mouse mutants of both $\mathrm{PC} 1 / 3$ and $\mathrm{PC} 2$ show multiple, partially overlapping defects in neuropeptide production and null mutations display increased lethality (PC1/3) and reduced growth (PC1/3 and PC2) (reviewed in Hoshino \& Lindberg, 2012).

The evolutionary successful and taxon rich insects are of particular value for understanding molecular mechanisms of neurosecretion. Insect neuropeptide signalling regulates essential biological processes such as behaviour, feeding and physiology, water balance, life cycle transitions and reproduction $[9,10]$. The neuroendocrine function of PC2 is conserved between mouse and Drosophila. Mutations of the fly's PC2 orthologue amontillado (amon) leads to impaired production of neuropeptides and is lethal $[7,11-13]$. However, the fly is a rather atypical model for PC-function as it has lost the ancestral PC1/3 gene $[7,11]$, while both are present in other insect genomes $[4,14]$. Hence, work in Drosophila is unlikely to reveal a comprehensive picture of ancestral PC-function in insects but rather is an example for a derived situation. This is particularly interesting because PC-processing-dependent neuropeptide signalling is thought to be involved in a multitude of biological processes. These include for example metabolic regulation by insulin-like peptides and adipokinetic hormone $[15,16]$. Insulin, among other peptides, is involved in the nutrient-dependent regulation of oocyte maturation (see for example Roy et al. 2018). In addition, neuropeptides are involved in the regulation of insect-specific evolutionary inventions like their growth and development through distinct larval and pupal stages separated by moults. The timing of moulting is regulated and depends on the growth rate. How the information of sufficient growth is integrated into the regulation of moulting is not fully understood. It is thought that a non-growing oxygen supply by the static tracheal system eventually triggers the moult $[18,19]$. Processed neuropeptides are likely involved in communicating this information to the moult triggering mechanism [20,21]. The mutant phenotype of the fly PC2 gene amon is developmental arrest during the first instar larval moult. The animal is not able to shed its old cuticle and becomes 'locked-in' [13]. However, nothing is known on PC-function in moulting and other processes in any other insect but the fly, and in particular, there is no functional information on $\mathrm{PC} 1 / 3$ function as this gene was lost in flies. One hypothesis would be that PC2 and PC1/3 in other insects act in a near redundant fashion and that PC2 would be able to largely compensate for loss of PC1/3, 
explaining how the loss of $\mathrm{PC} 1 / 3$ could have occurred in the fly lineage. In an opposing scenario, $\mathrm{PC} 1 / 3$ may have specific functions in other insects, which have become unnecessary in the fly due to changes in lifestyle or alterations in the regulatory pathways.

Here, we study the functional evolution of neuroendocrine processing by comparing the well-known but derived Drosophila situation to an insect with a conserved complement of enzymes and a different lifestyle. For this we use the red flour beetle Tribolium castaneum as a model system which is amenable to functional genetic work [22,23] and imaging of the CNS [24]. Furthermore, Tribolium has already been subject of some studies looking at the function of individual neuropeptides $[25,26]$. First, we confirmed that $P C 1 / 3$, which is evolutionary older than $P C 2$, is conserved in all insect groups except for dipterans. This genomic conservation is suggestive of a functional importance of retaining separate PC1/3 and PC2 orthologues. Indeed, we demonstrate that both Tribolium PC2 and PC1/3 have an essential function in maintaining metabolic balance and nutrient-dependent infertility. In larvae we found that the role of PC2 in larval-to-larval moulting is conserved between fly and beetle producing an amontillado-like locked-in phenotype. We also find PC1/3 backs up PC2 function in the moulting process as a double knockdown increases the penetrance of this phenotype. Unexpectedly, we characterize a novel and important individual role of $\mathrm{PC} 1 / 3$ at the larval stage: the gene is expressed in small cell groups of the CNS and is required for connecting growth to the moult cycle.

In conclusion, the parallel functions in adult beetles are comparable to the overlapping but not fully redundant functions of both these genes in vertebrates [8], whereas they both have additional insect specific functions at the larval stage. We discuss our findings on $\mathrm{PC} 1 / 3$ gene function in connection with current models on the interconnection of larval-to-larval moults with weight gain $[18,27]$ and discuss possible changes of growth and moult regulation in flies, in accordance with larval lifestyle, that may have made PC1/3-function dispensable in this lineage.

\section{Results}

\section{1) PC1/3 has a pre-bilaterian origin and is conserved in all insect groups except flies}

To understand the evolutionary history of the insect subtilisin-like protein convertases and to map patterns of gene loss, we scored genomes of selected species for presence or absence of these enzymes. There are seven PCs in mice and humans (PC1/3, PC2, PC5, PC4, PC6/PACE4, PC7 and furin), in addition to PCSK-9 and SKI-1, which also contain subtilisin-like catalytic domains but are membrane bound [28]. We surveyed well-assembled genomes representing major insect lineages in addition to other animal lineages. We then used 
identified PC sequences for gene family reconstruction (see gene tree figure $1 \mathrm{~A}$ ), using SKI-1 genes as an outgroup (PCSK9 was not found in any invertebrate genome).

When comparing different bilaterians it became evident that a set of four PCs is shared between disparate groups: PC1/3, PC2, PC7 and furin (see figure $1 \mathrm{~A}, \mathrm{~B}$ ). These were found in representatives of arthropods, molluscs and vertebrates, representing the major bilaterian lineages. Nematostella vectensis, belonging to the basally branching radial symmetric cnidarians, has a clear PC1/3 and PC7 orthologue, and one gene that groups with furins, but lacks PC2. In addition, there are six cnidarian PCs that do not group with any of the bilaterian convertases but form a group of their own (figure $1 \mathrm{~A}$ ). The verteberate furin proteins have undergone an expansion. Vertebrate PC4 and furin are closely related and both cluster with invertebrate furin1. The PC5 and PACE4 proteins also fall into the furin-group and are closely related to one another (figure $1 \mathrm{~A}$ ).

When compared to vertebrates, the PC protein family is more compact in insects, typically comprising PC1/3, PC2, furin1 and furin2 (with some additional lineage specific duplications of furin genes in hemipterans, lepidopterans and collembolans figure $1 \mathrm{~A}, \mathrm{~B})$. Two furin genes are the typical gene content in invertebrates as direct orthologues to the insect furins are present in the other invertebrate taxa as chelicerates, myriapods and the lophotrochozoan Lottia gigantea. In the myriapod Strigamia maritima, an arthropod with a conservative genome organization [29] a PC7 gene is present. It was lost, presumably independently, from spiders and all hexapod genomes. By contrast PC1/3 is conserved in all insect groups but no orthologues were detected in the dipteran genomes that we sampled (Drosophila melanogaster and Aedes aegyptii). In addition, BLAST searches for PC1/3 genes in the entire dipteran clade did not identify any orthologous sequence. Hence the factor was specifically lost in the lineage leading to the diptera. Similar to flies, Caeanorhabditis elegans, the other major invertebrate model organism, has lost its $\mathrm{PC} 1 / 3$ orthologue but only possesses a clear PC2 orthologue [30].

To score the degree of conservation of the Tribolium prohormone convertases, we compared the protein sequences. On the protein level the Tribolium neuroendocrine specific convertases $\mathrm{PC} 1 / 3$ and $\mathrm{PC} 2$ share moderate overall sequence similarity of $44 \%$. However, they have a near identical organization. An N-terminal signal peptide that directs the polypeptides to the neurosecretory pathway is followed by an S8-pro- and S8-peptidase catalytic domain exerting the enzymatic functions, and a P/HomoD regulatory domain (see 
figure 1C). This domain structure is conserved between the Tribolium and vertebrate neuroendocrine specific PCs [8]. A specific amino acid motif with a core of -RGD- has been identified within the active domain of vertebrate PC1/3 and PC2 and was shown to be required for sorting the vertebrate enzymes into the secretory vesicles [31]. This motif is present in Tribolium PC2 and in all other insect PC2 genes that we have looked at. However, the motif is absent from the PC1/3 protein of Tribolium and other insects, and from the myriapod Strigamia, but present in the cnidarian and chelicerate PC1/3 proteins.

\section{2) PC2 is expressed ubiquitously in cells of the nervous system whereas PC1/3 is restricted to identifiable cell populations}

PC1/3 has not been studied in any insect/arthropod before. Given that Tribolium contains the arthropod-typical set of two PCs, we asked, in how far PC1/3 and PC2 were expressed in different or overlapping cell groups. We performed RNA-in situ hybridization to detect specific expression of these genes in embryos and in the larval nervous system of Tribolium. We found that PC2 is expressed during late embryogenesis (NS 14, stages according to Biffar and Stollewerk 2014) in many cells of the head and trunk neuroectoderm (figure 2A). At a slightly later stage (NS 15), shortly before hatching, most neural cells of the developing central nervous system express PC2 (figure $2 \mathrm{~B}$ ). At the larval stage, $\mathrm{PC}$ expression is found in most cells of the nervous system (figure $2 \mathrm{C}$ ). A more intense expression is seen in the anterior medial brain area in larvae (arrow in Fig. $2 \mathrm{C}$ ) where the pars intercerebralis is located, a structure of the insect brain with known neuropeptidergic properties (see Strausfeld 2012). By contrast, we did not find any embryonic expression of PC1/3. Strikingly, expression in the larval nervous system was restricted to individual cells. In the suboesophageal ganglion we found two dorsally located cell groups. The anterior cluster comprised two cells (white arrow in Fig. 2D) and the more posterior normally contained 6 cells (white arrow in Fig. 2D). In one of four inspected nervous systems the posterior cluster comprised 8 cells, indicating some variability. Expression was also found laterally in the posterior brain (tritocerebrum; anterior white circle) where two cells on each side stain positive (arrowheads figure 2D). We found no signal in the PI or other cells of the protocerebrum, nor in the mid-thoracic ganglia.

\section{3) Both PC2 and PC1/3 are essential for survival and fertility of adult beetles}


Given that most arthropods have both $\mathrm{PC} 1 / 3$ and $\mathrm{PC} 2$ and given the different expression patterns in Tribolium we hypothesized that these enzymes would have important and at least partially different functions. In order to test this, we knocked down the genes individually and scored for different phenotype classes.

We first injected dsRNA targeting the respective factors into female pupae. The use of nonoverlapping fragments produced similar effects, indicating that the phenotypes were specific (also see supplementary material 3/ figure S1 for quantification of knock-down by qPCR).

We found that interfering with expression of $\mathrm{PC} 2$ and $\mathrm{PC} 1 / 3$, respectively led to severe reduction of survival rates compared to control-injected animals (figure $3 \mathrm{~A}$ ). Over the experimental time course of 41 days PC2-RNAi beetles $(n=100)$ had a total survival rate of only $20 \%$ whereas $48 \%$ of PC1/3-RNAi beetles survived, compared to $80 \%$ survival in the control (injected with dsRed-dsRNA, $n=30$ ). A repeat experiment confirmed the reduced viability of PC1/3- and PC2-dsRNA injected beetles when compared to a control set: after 30 days $44 \%$ of PC1/3-RNAi beetles survived, $38 \%$ of PC2-RNAi beetles and $67 \%$ of a control set ( $n=100 /$ each RNAi and $n=15 /$ control). Beetles of both knockdowns that died in the first few days after injection (up to day 5 , see figure $3 \mathrm{~A}$ ) typically showed signs of incomplete eclosion (figure $3 B, C$ ): the wings were not fully covering the abdomen. Abdomens were thin and of a dry appearance. The desiccation is possibly a result of the incomplete covering with the elytra. Also, later in the course of the experiment, beetles with fully elongated elytra showed sign of starvation and possibly desiccation (figure 3D, E). Wings were frequently half opened when dead, but since these beetles had gone through complete metamorphosis before, we assume that problems with metabolism and water balance led to this appearance and to their death. To test for effects on female fertility we mated females that were injected as pupae $(n=100$ for each gene) with untreated male beetles (ratio 5:1) in a bulk mating. We assessed their reproductive success at different time points by counting all eggs in a 24-hour period. We started from day 8 in order to include only adults that had successfully hatched. We found that the number of eggs was severely reduced in both treatments. From more than 10 eggs/female/24 $\mathrm{h}$ egg laying decreased to less than 2 eggs/female/24 $\mathrm{h}$ in PC2 and PC1/3 knockdown animals alike (see figure $4 \mathrm{~A}$ ). The small number of laid eggs developed at a normal rate to wildtype appearance. An independent repetition of this experiment confirmed a lasting reduced fertility of less than 1 egg/female/24 $\mathrm{h}$ over 30 days, compared to 7-11 eggs/female/24h in the control ( $n=100 /$ each RNAi and n=15/control, not shown). 
We then tested involvement of PC1/3 and PC2 function in male fertility by injecting male pupae and crossing 30 individual males to wildtype female beetles at a ratio of 1:5. Survival of males following injections was also reduced over the experimental time course of 14 days: PC1/3-RNAi: $36 \%$, PC2-RNAi: $42 \%$, dsRed-ctrl.: 86\%. We did find significantly reduced fertility of PC1/3 RNAi males and even more severely in PC2 RNAi beetles (figure 4B). In line with a partial recovery of injected beetles from the RNAi-effect, the reduction of male fertility grew less severe the more time had passed since injection. In addition, even though egg number was reduced significantly at all three timepoint, females mated to injected animals still laid a good number of eggs (figure 4B), and effects were generally milder than observed for female RNAi-beetles. The majority of these eggs were fertilized and developed into larvae at a rate not different to wildtype eggs (ctrl: $92 \%$ ( $n=106)$, PC1/3-RNAi: $93 \%(n=71)$, PC2-RNAi: $88 \%$ $(n=72))$. These numbers indicate for the presence of functioning sperm. Variation of egg number produced by individual matings were high and the death rate of the injected males was also high. The lowest number of eggs was usually obtained from males that would die shortly after. Together with the comparably moderate decrease of fertility, this suggests that the significant reduction of egg number following male treatment may have been a consequence of reduced overall fitness rather than a specific failure of sperm production or mating.

Taken together, we find a fitness related effect of PC1/3 and PC2 knockdown on male fertility and a strong and persistent effect on female egg production.

\section{4) Ovarial phenotypes underly the female infertility of PC1/3- and PC2-RNAi beetles}

To further understand the causes of female infertility we dissected and analysed ovaries of PC1/3- and PC2-RNAi beetles. First, we identified three classes of ovarial phenotypes (figures 5A,B): normal/wildtype-like, a so-called held-egg phenotype in which mature eggs accumulate in the oviduct without being laid, and a 'small-ovary' phenotype in which ovarioles and hence the whole ovaries are significantly smaller than wildtype ones. We found a comparable distribution of phenotypes in both knockdowns (figure 5C). Most ovaries were of the 'small' appearance (PC1/3: $62 \%, \mathrm{PC2}: 54 \%$ ), a small percentage (10\% in each knockdown) were classified as 'held-egg', while the remaining ovaries were wildtype-like. Even though we focussed on the timepoint of 14-15 days after injection, inspection of a small number of ovaries at 5 and 30 days revealed similar phenotypes. We further visualized the 
structure of the 'small-ovary' phenotype by fluorescently labelling them for actin (using phalloidin) and for nuclei (using DAPI). When comparing the 'small ovary' phenotype to wildtype ovaries (figure 5D) we found that all components of an ovariole were still present in the 'small ovary' phenotype (figure 5E). Like wildtype, they comprise a terminal germarium followed by egg chambers with oocytes of consecutive developmental stages (white arrowheads in Fig. 5D). In mild phenotypes the shapes of the ovarioles were not altered and the oocytes filled the whole egg-chambers, with their membrane lining the outer follicle cell sheet (figure $5 \mathrm{H}$ ) like in the wildtype. However, the most mature oocytes of this mild phenotype did not reach the same size as they would in wildtype-ovaries (white arrowheads in $\mathrm{D}$ and $\mathrm{E}$ ) and the lateral oviducts (red asterisks in Fig. $5 \mathrm{E}$ ) were not filled with eggs as in the wildtype. This indicates that these small ovaries phenotype were not capable of producing viable eggs. The more severe form of the 'small-ovary' phenotype (figure 5F) showed more slender proportions of ovarioles caused by the oocytes having a strongly reduced diameter. The oviducts are also not filled with mature eggs (red asterisks, figure 5F). Such defects could be primary effects of impeded neurosecretory signals or secondary due to starving of the animal. In order to distinguish these possibilities, we analysed wildtype animals, that had been starved for 4-10 days directly after eclosion. Indeed, PC knock-down ovaries looked similar to ovaries in beetles that suffered starvation (figure 5G, starved for 10 days, we did not find notable differences of the appearance of ovaries starved for $4,8,10$ or 12 days respectively). Note that severe and less severe forms of the 'small' ovary phenotypes were seen in both, PC2 and PC1/3 knockdowns. In the 'small-ovaries' of the severe form, as in the starved ovaries, the most mature oocytes remained small and did not have the typical oval shape. There were often gaps between the follicle cells and the oocyte membrane (red arrows in figure $5 \mathrm{I}$ and $\mathrm{J}$ ). We also observed a disintegration of some oocytes (red arrows in figure $5 F)$, which might be followed by reabsorption of the material by the follicle cells. No mature oocytes were located in the lateral oviducts of starved ovaries (red asterisks, figure 5G). Intriguingly, most of the severely reduced ovaries from both knockdowns had a slightly increased number of ovarioles: Tribolium ovaries normally comprise 10 ovarioles whereas the severely reduced ones often had 11-12 ovarioles (see for example figure 5F). We also occasionally found this in ovaries from starved beetles, but never in wildtype ovaries. Due to the small number of ovaries that were fully intact after dissection and staining procedures we were not able to quantify this effect. 
In summary we found that the dominant appearance in both PC1/3 and PC2 knockdown was the 'small ovary' phenotype in which oocytes failed to reach a mature size and form. This effect could be reproduced by depriving the beetles of food, indicating insufficient availability or distribution of nutrients to the oocytes in the RNAi-beetles. Note that the different classes of ovarial phenotypes taken together do not occur at a frequency that would fully explain the reduction in egg number. $28 \%$ of PC1/3- and $36 \%$ of PC2-RNAi ovaries were of wildtype appearance, whereas egg number was reduced to less than $20 \%$ in both knockdowns at 16 days. Therefore, we cannot exclude additional fitness-related or behavioural effects caused by interfering with the neuroendocrine system.

\section{5) Divergent functions of PC1/3 and PC2 in larval growth}

We wanted to know, in how far these enzymes were required for growth independently of metamorphosis or oogenesis. To this end we used larval RNAi to circumvent the infertility effect and test for effects of PC1/3 and PC2 gene knockdown on larval development. We also included a double knockdown of both factors into our analysis. Larvae were injected with the respective dsRNAs at 12 days after hatching (L4) and growth curves and moult cycles were recorded thereafter.

Wildtype and dsRed dsRNA-injected control animals showed a constant weight gain until they reached a weight of over $2 \mathrm{mg}$ (figure $6 \mathrm{~A}$ and see supplementary material 5/ figure S2 for growth curves of untreated and dsRed dsRNA control injected larvae). Then the larvae lost some weight as they stopped feeding in preparation for the larva to pupa transition (figure 6A, S2). By contrast, PC1/3 RNAi treated larvae stopped growth almost completely for several days (figure 6B). Some of the observed larvae showed near zero weight gain over a period of up to 30 days, and some of these individuals died prematurely (larval lethality was $50 \%$ ). Another half of the larvae did initially show a flat growth curve but entered a phase of increased weight gain at a later time and eventually reached a weight sufficient for larva to pupa transition. Due to the slowed growth the larval period was significantly prolonged in PC1/3 knockdown larvae: all control larvae had pupated by day 28 of development whereas $50 \%$ of PC1/3-RNAi specimens remained in the larval period beyond the age of 41 days. In an independent experiment we observed an individual PC1/3-dsRNA treated animal that had not pupated even after 3 months' time. Intriguingly, we observed supernumerary moults in the knock-down larvae (see below). 
PC2 dsRNA-injected larvae had a high mortality and half of them had already died on day one after injection (figure 6C), compared to zero deaths in control injected larvae at this timepoint. Unlike PC1/3 knockdown larvae PC2-RNAi larvae initially showed some weight gain, but still lagged behind the control sets (see figure 6C, E). All PC2-RNAi larvae eventually suffered rapid weight loss and were found dead shortly after. Larvae that were co-injected with PC1/3- and PC2-dsRNA, each at the same concentration of $1 \mu \mathrm{g} / \mu \mathrm{l}$ dsRNA targeting each gene showed a strongly reduced growth as known from the PC1/3 knockdown and a high lethality as observed in PC2 larvae (figure 6D) indicating additive function. Results of independent repeat experiments confirming the effect of PC1/3 and PC2 knockdown in larvae are shown in supplementary material S5/ figure S3.

In conclusion, these results showed that PC1/3 and PC2 are both required to maintain larval growth and survival. The different dynamics, however, indicated that both function independently at the larval stage.

\section{6) PC1/3 knock-down leads to the decoupling of growth from moulting}

In wildtype, larval growth and moulting are tightly coordinated and the number of larval instars is almost invariable. We wondered, how the reduced growth in PC1/3 and PC2 knockdown would affect the number of moults. Therefore, we plotted the moults on the average growth curves based on the above described experiments (Fig. 6E, green circles indicate the point in time where most animals moulted, the green dotted line marks the time range where moulting occurred). Most (5/8) PC2 injected larvae underwent one moult after some weight gain, but, accompanied by the high lethality, the majority of these larvae (4/5) did not go through the second moult cycle. Only a small proportion of PC1/3 and PC2 double RNAi animals did complete one moult (3/8), and none of them completed a second moult (figure $6 \mathrm{E})$. Unexpectedly, larvae treated with only PC1/3 dsRNA still went through larval moults despite strongly reduced growth (see above). They underwent the first two moults at similar points in time compared to control injections although there was almost no weight gain during that period (figure 6E). Some individuals went through moults without effective weight gain since the last moult (see supplementary material 6, table S4). The third moult was delayed by more than four days compared to the last larval-larval moult of wildtype. Interestingly, the PC1/3 knockdown larvae went through supernumerary moults while the increase in weight was minimal. 
Taken together, our experiments showed that a strong PC1/3-knockdown led to a strong inhibition of larval growth. Importantly, they revealed PC1/3 dependent peptide-processing as a novel molecular mechanism required for the coupling of larval moulting and growth.

7) amontillado-like locked-in phenotypes occur at a high penetrance in PC2 knockdown, and at a low frequency, in PC1/3 knockdown larvae

Mutants of the Drosophila PC2 orthologue amontillado, show a failure of the moulting process: the larva produces a new cuticle but is not able to shed its old cuticle and becomes "locked-in", which then leads to weight loss and death. Hence, Tribolium PC2-RNAi larvae were specifically screened for this phenotype once they had died. Indeed, a second cuticle was detected, unambiguously recognisable by the duplicated mandibles and terminal structures (figure 7A, $A^{\prime}$ ). By screening a number of deceased PC2 knockdown larvae (injected at 12 days) we found, that $75 \%(n=20)$ (see figure $7 B$ ) of them suffered from the locked-in phenotype. The remaining were very small when found dead, but no second cuticle was found. Somewhat surprisingly we also found this phenotype in PC1/3-RNAi larvae but at much lower penetrance. Survival of PC1/3 larvae was $50 \%$, and $26 \%$ of dead larvae showed a locked-in phenotype ( $\mathrm{n}$ total $=38, \mathrm{n}$ of inspected dead larvae $=19$ ), which gives a total penetrance of the phenotype of $13 \%$ (see figure 7B). The failure in the moulting process and the induction of supernumerary moults in PC1/3 RNAi indicate that the gene acts pleiotropically in multiple steps of moulting control. The results indicated that both $\mathrm{PC} 1 / 3$ and PC2 act in the moult pathway and that the other protease may able to partially rescue the process in the knockdown larvae. To test this, we screened larvae in which a double knockdown was performed (as shown in figure 6D) for a locked-in phenotype. We found that $100 \%$ of these larvae $(n=20)$ suffered from this phenotype (see figure 7B for comparison), supporting the hypothesis of collaborative function in this process. In addition, we evaluated which proportion of the RNAi-larvae were able to successfully complete one or two moults (figure 7C), before either dying of a locked-in phenotype, or other causes, or surviving to the pupal stage (PC1/3 RNAi larvae only). We found that $65 \%$ of PC2-RNAi larvae completed at least one moult cycle, compared to only $33 \%$ of the double knockdown larvae. None of the latter was able to complete a second moult cycle, whereas $15 \%$ of the PC2 single knockdown were able to do so. All PC1/3 knockdown larvae completed one moult and the large majority ( $89 \%$ ) also completed a second moult, reflecting the low penetrance of the phenotype in this 
knockdown. Taken together, PC1/3 and PC2 act together in the moult process and can partially compensate for one another's function while the contribution of PC2 to this process is bigger.

\section{Discussion}

Phylogenetic analysis of the insect prohormone convertase family identifies PC1/3 as evolutionary older but less conserved

Subtilisin-like prohormone convertases trace back to unicellular organisms [8,34] and signalling by peptide hormones is believed to be one of the earliest cell-to-cell communication system in animals [35]. We find that PC1/3-, PC2-, PC7-, and furin- orthologous genes are present in members of proto- and deuterostomes, i.e. both major bilaterian groups. Protostomes typically have furin1 and furin2, whereas PC4, PACE4 and PC5 are lineage specific duplications of furin that are only found in deuterostomes [14]. When including sequences of the non-bilaterian species Nematostella vectensis into the analysis we find orthologues of PC1/3 and PC7, and one furin, in addition to a number of PCs that do not fall into any of the clades but predominantly form a branch by themselves (referred to as basal PCs here). This is very similar to what was found in the sponge Amphimedon queens/andica [14] PC1/3, PC7 and furin trace back to a pre-bilaterian origin while PC2 is only found in Bilateria. Notably the phylogenetically younger gene PC2 appears to be more conserved in modern invertebrate lineages whereas PC1/3 has convergently been lost in some taxa, notably in the model organisms Drosophila and C. elegans [4,30]. Loss of PC7, which, among other functions, also is involved in neurohormone processing [36] has occurred even more frequently. It is missing in all insects and in chelicerates. Within insects, loss of $P C 1 / 3$ is restricted to the dipteran lineage only. Its absence goes in striking analogy to a reduction of neuroendocrine specific carboxypeptidases (CPS) from typically two (CPE and CPD) to only one gene in flies (CPD, Drosophila: silver) [37].

In summary, the loss of PC1/3 and CPE in the fly genome makes Drosophila a highly derived model for this process. Similarly, the PC gene complement is highly diverged in the other protostome model system C. elegans $[30,38]$. Given this situation, Tribolium represents an excellent complementary protostome model with typical gene complement and a growing toolkit for studying gene function. 


\section{A new PC1/3 expressing cell population of the beetle's suboesophageal ganglion}

We report a broad expression of PC2 in the embryonic neuroectoderm and the larval CNS of Tribolium, which is similar to PC2-expression during Drosophila embryogenesis and in many secretory neurons of the fly nervous system [7,39]. Expression of PC2-orthologues in invertebrate nervous systems and/or neuroendocrine organs has also been reported from a shrimp, a centipede, C. elegans and the polychaete worm Platynereis [38,40-42], underlining the conserved nature of this gene.

By contrast, $\mathrm{PC} 1 / 3$ expression or function has to date not been investigated in any invertebrate. PC1/3 expression at the larval stage is very specific to some cells, most of which are located in the suboesophageal ganglion. Some cells of the Drosophila suboesophageal ganglion have a unique neuropeptide expression profile including the perivisceroscokinins capa and hugin, fmrf-a and leucokinin [16]. It is however yet unclear if any of these groups can potentially be homologised to the PC1/3 cells in Tribolium. The presence of this specific set of cells that express $\mathrm{PC} 1 / 3$ suggests that they have a specific role in neuroendocrine regulation. Both their developmental origin and their function require further studies.

\section{Essential and shared role of PC2 and PC1/3 in maintaining metabolic balance parallels function of both genes in vertebrates}

Carbohydrate metabolism in insects is mainly regulated by two neuropeptides, adipokinetic hormone (AKH), which works analogous to vertebrate glucagon: its action mobilises sugars and lipids from the fat body and thus increases circulating sugar levels in the haemolymph [43-46]. Besides having multiple function in insects, insulin-like peptides are able to decrease blood sugar levels by directing the carbohydrates to the cells where they are required (Antonova et al., 2012). Both AKH and insulin precursors contain constitutive prohormone convertase cleavage sites and Drosophila amon has been shown to be required for AKH production in cells of the corpora cardiaca [11]. The observed poor metabolic condition of adult beetles deficient of normal PC1/3 or PC2 levels is likely to be a result of impaired AKH and/or insulin signalling. In addition, they appear to suffer from desiccation. Water balance and metabolism are closely intertwined in insects [44] and impairment of normal sugar homeostasis would impact the animals ability to retain metabolic water. In addition there are also a number of neuropeptides that control water balance by acting as diuretic hormones, 
such as DH31 and DH37/47 [44,47]. Interestingly, ADFs-genes (antidiuretic factors) have expanded to five orthologues in Tribolium, which can be interpreted as an adaptation allowing the extreme dry tolerance of this beetle $[47,48]$. All these factors are likely to be affected by knockdown of PC1/3 and PC2, and we interpret the phenotype as a broad failure of multiple physiological processes, with the most prominent symptoms potentially obscuring more subtle defects.

Within vertebrates, PC1/3 and PC2 function is best studied in mice. Knockout of each factor leads to multiple defects in neuropeptide processing. Mutant models of PC1/3 show high developmental and neo-natal death rates, and surviving individual are runted $[49,50]$. Similarly, the phenotype of PC2 is reduced growth and chronic hypoglycaemia caused by a lack glucagon and pro-insulin processing [51-53]. In mice PC1/3 and PC2 partially act in the same pathways, and many neuropeptide precursors are processed by both enzymes [8]. Similar to our findings in a beetle, mutants of both enzymes show severe defects (with PC1/3 being the more dramatic one) and there is no full redundancy between these enzymes.

\section{PC-dependent neuropeptide signalling is required for female oocyte maturation in a nutrient dependent fashion and for male reproductive fitness}

Most female insects deposit large amounts of nutrients, especially yolk proteins, into the developing oocyte. Our results clearly show that inhibition of both proteases disrupts this process and an insufficient amount of nutritive substances is deposited to the oocytes. This may on the one hand be caused by the inability of the injected beetles to maintain sugar and lipid- homeostasis. On the other hand, neuropeptides are directly involved in regulating vitellogenesis [17]. In some insects, juvenile hormone $(\mathrm{JH})$ secretion from the corpora allata positively regulates this process and different neuropeptides, such as allatotropin, allatostatins and ecdysis triggering hormone are involved in regulating $\mathrm{JH}$-synthesis [54]. In Tribolium specifically, it has been shown that insulin like peptides (ILPs), in response to JH and nutrient availability, and together with the transcription factor Tc-FoxO, are required for active vitellogenin transcription [55]. Hence, the severe effects on oocyte maturation that we see in PC1/3- and PC2-RNAi beetles most likely reflect physiological failures in both, the neuropeptide-dependent regulation of fat and sugar metabolism, and the specific regulated distribution of yolk proteins to the oocytes. 
Male fertility and the process of spermatogenesis is far less studied than female oogenesis. Naturally the production of sperm is a process that is less nutrient intense than oocyte production. Therefore, the effect on male fecundity might be less severe because a poor metabolic condition of the beetles plays a minor role here. However, insect peptide hormones have been implicated in the regulation of testes development and sperm number [56]. In addition, neuropeptides are involved in regulating male sexual behaviour [9]. Notably, the precursors of two peptides, NPF and PDF, that play major roles in regulating male reproductive behaviour in flies [57,58] are missing from the Tribolium genome [47]. This indicates another divergence of the neuroendocrine systems within insects, which merits further study.

\section{Collaboration of both prohormone convertases in moulting process itself with a more prominent role of $\mathrm{PC} 2$ precedes redundancy of $\mathrm{PC} 1 / 3$ in this process}

The most prominent mutant phenotype of the Drosophila PC2 homologue amon is developmental arrest during the first larval instar moult ("locked-in" phenotype) due to an incapability to complete ecdysis (Rayburn et al., 2009). PC2 knockdown in Tribolium produced the same phenotype at a high penetrance showing the conserved involvement of this factor in a number of involved processes. First, functional analysis of moulting fluid proteins by Zhang et al. 2014 [59] in Tribolium larvae frequently produced phenotypes in which cuticle shedding was impaired, similar to the prohormone convertase phenotypes we describe. Although subtilisin-like protein convertases do not appear to be abundant in the fluid [59], PC-processed neuropeptides may be involved in the initiation of moulting fluid secretion. Second, the partial digestion of the old cuticle by moulting fluid is followed by specific moulting behaviour which eventually leads to the breaking of the old cuticle [27]. Several neuropeptides have been implicated in this process. Ecdysis triggering hormone (ETH), which is secreted from the brain, starts the process. Essential for the interpretation of the ETH-signal are CCAP- (crustacean cardioactive peptide) neurons in the central nervous system [60]. Both these factors require prohormone convertase protease processing. Hence failure of these two peptide signals is in theory sufficient for explaining the locked-in phenotype. We found a minor role for $\mathrm{PC} 1 / 3$ in the moulting process as well, probably reflecting by a partially overlapping pattern of neuropeptide processing. 


\section{PC1/3 promotes growth and has an unexpected role in coupling growth and larval moults}

With regards to larval growth, PC1/3 and PC2 knockdown have different effects. Following $\mathrm{PC1}$ /3-RNAi, growth is severely reduced, down to zero-growth in some individuals. PC2-RNAi larvae initially show a better growth rate but then undergo severe and rapid weight loss, and then die. Dramatically reduced weight is also characterizing Drosophila amontillado- (PC2-) mutant larvae [13]. Inhibited growth in PC1/3 phenotypes goes along with supernumerary moults and a prolonged larval period. Reduced growth in larvae can occur naturally by limited food supply, for example when a culture is overcrowded. It can also lead to prolonged larval development accompanied by supernumerary moults (see Kingsolver, 2007; Ruang-Rit \& Park, 2018). Insect larvae require a species-specific critical mass to induce the hormonal cascade initiating metamorphosis [63]. In Tribolium metamorphosis is normally initiated when the larvae reach a weight of app. $2 \mathrm{mg}$ (see Chafino et al., 2019). The additional moults observed in Tribolium PC1/3-RNAi larvae may hence be a secondary effect of the reduced weight gain. In insects larval growth is promoted by insulin-like peptides $[15,27,65]$ and the Tribolium genome contains a total of four insulin-like peptide precursor genes, which require processing to produce the mature bioactive peptides [47]. Hence, insulin-like peptides are likely candidates to be subject to $\mathrm{PC} 1 / 3$ processing and defective insulin signalling may explain the reduced growth following PC1/3 RNAi. In vertebrates pro-insulin is targeted by both PC1/3 and PC2 [8].

The most intriguing effect of $P C 1 / 3$ transcript depletion is that larval moults occur after minimal weight gain, and in some individual without any positive growth in the intermoulting period. Normally the non-growing cuticle limits further increase in mass and moulting is necessary to allow for additional growth. Therefore, moulting without growth is not advantageous and both growth and moulting are interdependent in wildtype conditions $[20,27]$. While there is no specific weight that has been associated with the initiation of a larval moult, some degree of growth must occur for the larvae to reach a threshold size that allows for a moult $[18,27]$. The mechanism by which larvae decide that growth was sufficient is not fully understood. One theory that appears be able to explain the PC1/3 phenotype solely as a reduction of growth is the so-called 'leaky prothoracic gland' hypothesis. According to this a constant but low level secretion of ecdysone from the prothoracic gland would ultimately lead to a threshold level of circulating hormone sufficient to induce moulting (see Nijhout et al., 2014). However, within this scenario moulting would only occur with significant 
delay, which is not the case in the growth-inhibited PC1/3 RNAi-larvae whose moult rhythm is only slightly shifted when compared to wildtype larvae. Therefore, we prefer an alternative explanation based on the hypoxia hypothesis of larval moult induction: as the tracheal system cannot not grow between moults, oxygen is suggested to become the limiting factor for the growing larva and hypoxia is then assumed to act as the trigger to induce moults $[18,20,66]$. However, whereas molecular mechanisms of hypoxia sensing are well understood in Drosophila [67-69], it is not known how the information of hypoxia is relayed and leads to an interpretation at the organism level [66]. Our data indicates that, in insects other than flies, the mechanism involves neuropeptide processing by $\mathrm{PC} 1 / 3$, providing a starting point to understand this molecular communication.

\section{Conclusions}

The independent loss of PC1/3 in both, fly and nematode genomes, might have indicated that it is rather dispensable and may function fully redundantly with its orthologue PC2. By contrast, our experiments showed that $\mathrm{PC} 1 / 3$ has essential functions in the beetle. At the adult stage it works in parallel with its paralogue PC2, but both are not redundant as individual knockdowns cause deleterious, presumably predominantly metabolic phenotypes. The individual role of PC1/3 in regulating growth and moulting at the larval stage is also essential, hence loss of PC1/3in flies is not preceded by redundancy of the factor in other insects. In line with genomic conservation, its function might well be conserved across insect groups and in flies the interplay of growth and moulting must be co-ordinated by an altered system. An analysis of PC1/3 targets in the beetle based on cell-specific expression analysis and quantitative peptidomic approaches, together with functional testing, have the potential to characterize neuropeptide signalling systems involved in the regulation of moulting dependent on growth.

\section{Methods}

\section{Genomic, phylogenetic and gene structure analysis}

In order to comprehensively analyse the presence and absence of subtilisin-like protein convertases in insects we searched individual genomes of representatives of all major insect groups for which 
genomic information is available. We also included other invertebrate and vertebrate groups into our analysis (see supplementary table S1 for all species and accession numbers). Identified sequences were classified by gene tree reconstruction using MAFFT alignment [70], sequence trimming using Trimal/gappyout [71] and tree reconstruction using FastTree2 [72] with a JTT-model [73]. Lastly, we performed NCBI-Blast searches on phylum level to confirm that the detected presence-/absencesituation is typical for the whole respective insect group. To identify structural properties of the proteins the signal peptide prediction site SignalP-5.0 (CBS, Technical University of Denmark) and the domain search InterPro (EMBL-EBI) were used.

\section{RNA in situ hybridisation to detect gene expression}

Gene fragments of PC1/3 and PC2 were PCR-amplified from cDNA using gene specific primers (see primer list in supplementary table S2) and inserted into a pJet1.2 cloning vector. Probes were synthesized using the 5X Megascript T7 kit (Ambion) according to the standard protocol. Embryo fixation and RNA-in situ hybridization was performed as previously described [74]. Nervous systems were dissected from mid-stage larvae and fluorescent in situ hybridization was performed as described in Hunnekuhl et al. (2020), also including staining with an anti-Synapsin antibody (mouse, DHSB Hybridoma Bank) and a DAPI for nuclei (4,6-diamidino-2-phenylindole; Sigma-Aldrich). Specimens were mounted in Vectashield (Vector labs) and a Zeiss LSM980 confocal microscope was used for image acquisition. Image stacks were analysed using FIJI [75].

\section{DsRNA injections and qPCR assay}

Two non-overlapping fragments of each gene were PCR-amplified (see primer list in table S2) and then used for dsRNA synthesis (see supplementary material 2 for details). In addition, a dsRed-dsRNA was generated for control injections (see supplementary material 2). Quantitative PCR confirmed dsRNA efficiency and target specificity (see supplementary material 3, figure S1). For the qPCR experiments RNA samples of WT, dsRed-, PC1/3- and PC2-dsRNA injected larvae were isolated using a Quick-RNA Tissue/Insect Kit including on-column DNAsel digest (Zymo Research), and subsequently reverse transcribed to cDNA. Primer pairs were designed with at least one primer of each combination spanning a splice site (supplementary table S3) and excluding the part of the gene used for dsRNA production. Primers amplifying the ribosomal protein RPS3 were used as a standard. The qPCR experiments were run using a C1000 thermal cycler with a CFX96 detection system (Bio-Rad). See supplementary material S3 for further details.

All beetles used for injections and as controls were of the San Bernadino (SB) wildtype strain. Pupal injections were carried out as described in Posnien et al. 2009 [23]. Larval injections were performed 
by first immobilising the larvae by cooling them in dish placed on ice. They were then mounted on pre-cooled petri-dishes containing a layer of $2 \%$ agar in tab water. Lining up the larvae along an edge in the agar prevented them from moving during the injection process. Both larval and pupal injections were carried out at a $1 \mu \mathrm{g} / \mu \mathrm{l}$ concentration unless stated otherwise.

\section{Ovarial dissections and staining}

Ovaries were dissected out of the abdomina from 14-15-day old females. A subset of ovaries classified into the respective phenotypes were further processed for fluorescent staining. The ovarioles were individually freed from their muscular lining with ultra-fine dissection needles. Ovaries were then fixed in $4 \%$ formaldehyde/ PBT for $30 \mathrm{~min}$ and stained overnight in Phalloidin-A488 (Sigma) at a concentration of 1:40 (v/v in PBT). Specimens were then washed $4 \times 10 \mathrm{~min}$ in PBT and stained with DAPI (v/v 1/1000 of a $1 \mu \mathrm{g} / \mu$ l stock solution in PBT) for 20 min. After 2 more PBT washes ovaries were mounted and imaged in the same way described for the stained nervous systems above.

\section{Survival, fertility and growth data acquisition}

Following pupal RNAi, survival rates were measured by counting living individuals at 3-4-day intervals. In order to measure fertility rates all injected females were crossed with wildtype males (ratio 5:1) in a bulk mating and the total number of eggs laid in a 24-hour period was counted. The male fertility assay was set up by mating injected males to wildtype females at a ratio of 1:5. Each of these malefemale combinations was kept on flour in a separate petri-dish. The egg number of 24-hour periods was counted from each individual cross and was averaged over the total number of males used. Statistical significance was tested using 1-tailed T-test type 3 applying conventional significance thresholds of * $(\mathrm{P}<0.05),{ }^{* *}(\mathrm{P}<0.01)$ and $* * *(\mathrm{P}<0.001)$.

For the generation of growth curves wildtype control- and injected larvae were kept individually on 12-well plates with a non-limiting supply of flour. Larvae were checked at intervals of 2-3 days; moults were recorded, and larvae were weighed using a high precision scale (model CP225D, Sartorius).

\section{Larval cuticle preparations}

Dead larvae were picked from the culture dishes and washed in $\mathrm{dd}_{2} \mathrm{O}$, followed by a $30 \mathrm{sec}$ wash in $50 \%$ bleach (Klorix) and another 2-3 washes in $\mathrm{ddH}_{2} \mathrm{O}$. Specimens were then mounted in Hoyer's

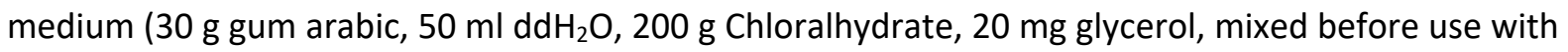
lactic acid at a ratio of $1: 1$ ) and put to $60^{\circ} \mathrm{C}$ overnight, and then analysed using a standard light microscope. 


\section{Declarations}

Ethics approval and consent to participate

Not applicable.

\section{Consent for publication}

Not applicable.

\section{Availability of data and materials}

The datasets generated during and analysed during the current study are available in the figshare repository: https://doi.org/10.6084/m9.figshare.12981818.v1.

\section{Competing interests}

The authors declare that they have no competing interests.

\section{Funding}

The work was partially funded by a DFG-project (TE 1380/1-1) grant to VHS. We acknowledge support by the Open Access Publication Funds of the Göttingen University.

Author contributions: VSH developed the project, SF and VSH carried out the experiments, VSH wrote the paper. Both authors read and approved of the final manuscript.

Acknowledgements: Firstly, we thank Gregor Bucher for sharing lab infrastructure, for discussions on the experiments and for valuable comments on the manuscript. We also thank Claudia Hinners for technical assistance, Magdalena Schacht and Janna Siemanowski for supporting the project at an early stage, Salim Hakeemi for introducing us to Tribolium ovarial dissections, and Rolando Rivera-Pomar and Ernst Wimmer for discussions on the experiments.

\section{Authors' information}

Not applicable. 


\section{References}

1. Arendt D. The evolution of cell types in animals: Emerging principles from molecular studies. Nat Rev Genet. 2008;9:868-82.

2. Guijarro-Clarke C, Holland PW, Paps J. Widespread patterns of gene loss in the evolution of the animal kingdom. Nat Ecol Evol [Internet]. Springer US; 20. Available from:

http://dx.doi.org/10.1038/s41559-020-1129-2

3. Fernández R, Gabaldón T. Gene gain and loss across the metazoan tree of life. Nat Ecol Evol. 2020;

4. Tessmar-Raible K. The evolution of neurosecretory centers in bilaterian forebrains: Insights from protostomes. Semin Cell Dev Biol. 2007;18:492-501.

5. Elphick MR, Mirabeau O, Larhammar D. Evolution of neuropeptide signalling systems. J Exp Biol [Internet]. 2018;221:jeb151092. Available from: http://jeb.biologists.org/lookup/doi/10.1242/jeb.151092

6. Jekely G. Global view of the evolution and diversity of metazoan neuropeptide signaling. Proc Natl Acad Sci [Internet]. 2013;110:8702-7. Available from:

http://www.pnas.org/cgi/doi/10.1073/pnas.1221833110

7. Wegener $\mathrm{C}$, Herbert $\mathrm{H}$, Kahnt J, Bender M, Rhea JM. Deficiency of prohormone convertase dPC2 (AMONTILLADO) results in impaired production of bioactive neuropeptide hormones in Drosophila. J Neurochem. 2011;118:581-95.

8. Hoshino A, Lindberg I. Peptide Biosynthesis: Prohormone Convertases $1 / 3$ and 2 [Internet]. Colloq. Ser. Neuropeptides. 2012. Available from:

http://www.morganclaypool.com/doi/abs/10.4199/C00050ED1V01Y201112NPE001

9. Schoofs L, De Loof A, Van Hiel MB. Neuropeptides as Regulators of Behavior in Insects. Annu Rev Entomol [Internet]. 2017;62:35-52. Available from:

http://www.annualreviews.org/doi/10.1146/annurev-ento-031616-035500

10. Kim SM, Su C-Y, Wang JW. Neuromodulation of Innate Behaviors in Drosophila. Annu Rev Neurosci [Internet]. 2017;40:327-48. Available from:

http://www.annualreviews.org/doi/10.1146/annurev-neuro-072116-031558

11. Rhea JM, Wegener $\mathrm{C}$, Bender $\mathrm{M}$. The proprotein convertase encoded by amontillado (amon) is required in Drosophila corpora cardiaca endocrine cells producing the glucose regulatory hormone AKH. PLoS Genet. 2010;6:2.

12. Rayburn LYM, Rhea J, Jocoy SR, Bender M. The proprotein convertase amontillado (amon) is required during Drosophila pupal development. Dev Biol [Internet]. Elsevier Inc.; 2009;333:48-56. Available from: http://dx.doi.org/10.1016/j.ydbio.2009.06.021

13. Rayburn LYM, Gooding HC, Choksi SP, Maloney D, Kidd AR, Siekhaus DE, et al. amontillado, the Drosophila homolog of the prohormone processing protease $\mathrm{PC2}$, is required during embryogenesis and early larval development. Genetics. 2003;163:227-37. 14. Hammond MJ, Wang T, Cummins SF. Characterisation of early metazoan secretion through associated signal peptidase complex subunits, prohormone convertases and carboxypeptidases of the marine sponge (Amphimedon queenslandica). PLoS One. 2019;14:1-15.

15. Antonova Y, Arik AJ, Moore W, Riehle MA, Brown MR. Insulin-like Peptides: Structure, Signaling, and Function. Insect Endocrinol [Internet]. Elsevier; 2012. p. 63-92. Available from: http://dx.doi.org/10.1016/B978-0-12-384749-2.10002-0

16. Nässel DR, Winther ÅME. Drosophila neuropeptides in regulation of physiology and behavior. Prog Neurobiol. 2010;92:42-104. 
17. Roy S, Saha TT, Zou Z, Raikhel AS. Regulatory Pathways Controlling Female Insect Reproduction. Annu Rev Entomol [Internet]. 2018;63:489-511. Available from: http://www.annualreviews.org/doi/10.1146/annurev-ento-020117-043258

18. Callier V, Nijhout HF. Control of body size by oxygen supply reveals size-dependent and size-independent mechanisms of molting and metamorphosis. Proc Natl Acad Sci U S A. 2011;108:14664-9.

19. Greenlee KJ, Harrison JF. Development of respiratory function in the American locust Schistocerca americana: II. Within-instar effects. J Exp Biol. 2004;207:509-17.

20. Nijhout HF, Riddiford LM, Mirth C, Shingleton AW, Suzuki Y, Callier V. The developmental control of size in insects. Wiley Interdiscip Rev Dev Biol. 2014;3:113-34.

21. O'Brien MA, Taghert PH. A peritracheal neuropeptide system in insects: Release of myomodulin-like peptides at ecdysis. J Exp Biol. 1998;201:193-209.

22. Gilles AF, Schinko JB, Averof M. Efficient CRISPR-mediated gene targeting and transgene replacement in the beetle Tribolium castaneum. Development [Internet]. 2015;142:2832-9. Available from: http://dev.biologists.org/cgi/doi/10.1242/dev.125054

23. Posnien N, Schinko J, Grossmann D, Shippy TD, Konopova B, Bucher G. RNAi in the red flour beetle (Tribolium). Cold Spring Harb Protoc. 2009;4:1-7.

24. Hunnekuhl VS, Siemanowski J, Farnworth MS, He B, Bucher G. Immunohistochemistry and Fluorescent Whole Mount RNA In Situ Hybridization in Larval and Adult Brains of Tribolium. Methods Mol Biol. 2020. p. 233-51.

25. Arakane Y, Li B, Muthukrishnan S, Beeman RW, Kramer KJ, Park Y. Functional analysis of four neuropeptides, EH, ETH, CCAP and bursicon, and their receptors in adult ecdysis behavior of the red flour beetle, Tribolium castaneum. Mech Dev [Internet]. Elsevier Ireland Ltd; 2008;125:984-95. Available from: http://dx.doi.org/10.1016/j.mod.2008.09.002

26. Aikins MJ, Schooley DA, Begum K, Detheux M, Beeman RW, Park Y. Vasopressin-like peptide and its receptor function in an indirect diuretic signaling pathway in the red flour beetle. Insect Biochem Mol Biol. 2008;38:740-8.

27. Belles X. Molting: the basis for growing and for changing the form. Insect Metamorph. 2020. p. 199-215.

28. Seidah NG, Sadr MS, Chrétien M, Mbikay M. The multifaceted proprotein convertases: Their unique, redundant, complementary, and opposite functions. J Biol Chem. 2013;288:21473-81.

29. Chipman AD, Ferrier DEK, Brena C, Qu J, Hughes DST, Schröder R, et al. The First Myriapod Genome Sequence Reveals Conservative Arthropod Gene Content and Genome Organisation in the Centipede Strigamia maritima. PLoS Biol. 2014;12.

30. Poole CB, Jin J, McReynolds LA. Subtilisin-like proteases in nematodes. Mol Biochem Parasitol. 2007;155:1-8.

31. Lusson J, Benjannet S, Hamelin J, Savaria D, Chrétien M, Seidah NG. The integrity of the RRGDL sequence of the proprotein convertase PC1 is critical for its zymogen and C-terminal processing and for its cellular trafficking. Biochem J. 1997;326:737-44.

32. Biffar $L$, Stollewerk A. Conservation and evolutionary modifications of neuroblast expression patterns in insects. Dev Biol [Internet]. Elsevier; 2014;388:103-16. Available from: http://dx.doi.org/10.1016/j.ydbio.2014.01.028

33. Strausfeld NJ. Arthropod Brains: Evolution, functional elegance, and historical significance. The Belknap Press of Harvard University Press; 2012.

34. Seidah NG, Hamelin J, Mamarbachi M, Dong W, Tadros H, Mbikay M, et al. cDNA structure, tissue distribution, and chromosomal localization of rat PC7, a novel mammalian 
proprotein convertase closest to yeast kexin-like proteinases. Proc Natl Acad Sci U S A. 1996;93:3388-93.

35. Achim K, Arendt D. Structural evolution of cell types by step-wise assembly of cellular modules. Curr Opin Genet Dev [Internet]. Elsevier Ltd; 2014;27:102-8. Available from: http://dx.doi.org/10.1016/j.gde.2014.05.001

36. Wetsel WC, Rodriguiz RM, Guillemot J, Rousselet E, Essalmani R, Kim IH, et al. Disruption of the expression of the proprotein convertase PC7 reduces BDNF production and affects learning and memory in mice. Proc Natl Acad Sci U S A. 2013;110:17362-7.

37. Pauls D, Hamarat Y, Trufasu L, Schendzielorz TM, Gramlich G, Kahnt J, et al. Drosophila carboxypeptidase $D$ (SILVER) is a key enzyme in neuropeptide processing required to maintain locomotor activity levels and survival rate. Eur J Neurosci. 2019;50:3502-19. 38. Husson SJ, Clynen E, Baggerman G, Janssen T, Schoofs L. Defective processing of neuropeptide precursors in Caenorhabditis elegans lacking proprotein convertase 2 (KPC2/EGL-3): Mutant analysis by mass spectrometry. J Neurochem. 2006;98:1999-2012. 39. Siekhaus DE, Fuller RS. A role for amontillado, the Drosophila homolog of the neuropeptide precursor processing protease PC2, in triggering hatching behavior. J Neurosci [Internet]. 1999;19:6942-54. Available from:

http://www.ncbi.nlm.nih.gov/pubmed/10436051

40. Hunnekuhl VS, Akam M. An anterior medial cell population with an apical-organ-like transcriptional profile that pioneers the central nervous system in the centipede Strigamia maritima. Dev Biol [Internet]. Elsevier; 2014;396:136-49. Available from:

http://dx.doi.org/10.1016/j.ydbio.2014.09.020

41. Tangprasittipap A, Chouwdee S, Phiwsaiya K, Laiphrom S, Senapin S, Flegel TW, et al. Structure and expression of a shrimp prohormone convertase 2. Gen Comp Endocrinol [Internet]. Elsevier Inc.; 2012;178:185-93. Available from: http://dx.doi.org/10.1016/j.ygcen.2012.05.005

42. Tessmar-Raible K, Raible F, Christodoulou F, Guy K, Rembold M, Hausen H, et al. Conserved Sensory-Neurosecretory Cell Types in Annelid and Fish Forebrain: Insights into Hypothalamus Evolution. Cell. 2007;129:1389-400.

43. Lee G, Park JH. Hemolymph sugar homeostasis and starvation-induced hyperactivity affected by genetic manipulations of the adipokinetic hormone-encoding gene in Drosophila melanogaster. Genetics. 2004;167:311-23.

44. Gäde G. Regulation of Intermediary Metabolism and Water Balance of Insects By Neuropeptides. Annu Rev Entomol. 2004;49:93-113.

45. Stone J V., Mordue W, Batley KE, Morris HR. Structure of locust adipokinetic hormone, a neurohormone that regulates lipid utilisation during flight. Nature. 1976;263:207-11.

46. Isabel G, Martin JR, Chidami S, Veenstra JA, Rosay P. AKH-producing neuroendocrine cell ablation decreases trehalose and induces behavioral changes in Drosophila. Am J Physiol Regul Integr Comp Physiol. 2005;288:531-8.

47. Li B, Predel R, Neupert S, Hauser F, Tanaka Y, Cazzamali G, et al. Genomics, transcriptomics, and peptidomics of neuropeptides and protein hormones in the red flour beetle Tribolium castaneum. Genome Res. 2008;18:113-22.

48. Brown SJ, Shippy TD, Miller S, Bolognesi R, Beeman RW, Lorenzen MD, et al. The red flour beetle, Tribolium castaneum (Coleoptera): A model for studies of development and pest biology. Cold Spring Harb Protoc. 2009;4.

49. Zhu X, Zhou A, Dey A, Norrbom C, Carroll R, Zhang C, et al. Disruption of PC1/3 expression in mice causes dwarfism and multiple neuroendocrine peptide processing 
defects. Proc Natl Acad Sci [Internet]. 2002;99:10293-8. Available from:

http://www.pnas.org/cgi/doi/10.1073/pnas.162352599

50. Mbikay M, Croissandeau G, Sirois F, Anini Y, Mayne J, Seidah NG, et al. A targeted deletion/insertion in the mouse Pcsk1 locus is associated with homozygous embryo preimplantation lethality, mutant allele preferential transmission and heterozygous female susceptibility to dietary fat. Dev Biol. 2007;306:584-98.

51. Furuta M, Zhou A, Webb G, Carroll R, Ravazzola M, Orci L, et al. Severe Defect in Proglucagon Processing in Islet A-cells of Prohormone Convertase 2 Null Mice. J Biol Chem. 2001;276:27197-202.

52. Furuta M, Carroll R, Martin S, Swift HH, Ravazzola M, Orci L, et al. Incomplete processing of proinsulin to insulin accompanied by elevation of Des-31,32 proinsulin intermediates in islets of mice lacking active PC2. J Biol Chem. 1998;273:3431-7.

53. Furuta M, Yano H, Zhou A, Rouillé Y, Holst JJ, Carroll R, et al. Defective prohormone processing and altered pancreatic islet morphology in mice lacking active SPC2. Proc Natl Acad Sci U S A. 1997;94:6646-51.

54. Lenaerts C, Monjon E, Van Lommel J, Verbakel L, Vanden Broeck J. Peptides in insect oogenesis. Curr Opin Insect Sci [Internet]. Elsevier Inc; 2019;31:58-64. Available from: https://doi.org/10.1016/j.cois.2018.08.007

55. Sheng Z, Xu J, Bai H, Zhu F, Palli SR. Juvenile hormone regulates vitellogenin gene expression through insulin-like peptide signaling pathway in the red flour beetle, Tribolium castaneum. J Biol Chem. 2011;286:41924-36.

56. Marciniak P, Urbański A, Kudlewska M, Szymczak M, Rosiński G. Peptide hormones regulate the physiological functions of reproductive organs in Tenebrio molitor males. Peptides [Internet]. Elsevier Inc.; 2017;98:35-42. Available from:

http://dx.doi.org/10.1016/j.peptides.2016.06.006

57. Kim WJ, Jan LY, Jan YN. A PDF/NPF neuropeptide signaling circuitry of male Drosophila melanogaster controls rival-induced prolonged mating. Neuron [Internet]. Elsevier Inc.; 2013;80:1190-205. Available from: http://dx.doi.org/10.1016/j.neuron.2013.09.034

58. Lee G, Bahn JH, Park JH. Sex- and clock-controlled expression of the neuropeptide F gene in Drosophila. Proc Natl Acad Sci U S A. 2006;103:12580-5.

59. Zhang J, Lu A, Kong L, Zhang Q, Ling E. Functional analysis of insect molting fluid proteins on the protection and regulation of ecdysis. J Biol Chem. 2014;289:35891-906.

60. Mena W, Diegelmann S, Wegener C, Ewer J. Stereotyped responses of drosophila peptidergic neuronal ensemble depend on downstream neuromodulators. Elife. 2016;5:22. 61. Ruang-Rit K, Park Y. Endocrine system in supernumerary molting of the flour beetle, Tribolium freemani, under crowded conditions. Insect Biochem Mol Biol [Internet]. Elsevier Ltd; 2018;101:76-84. Available from: https://doi.org/10.1016/j.ibmb.2018.08.002

62. Kingsolver JG. Variation in growth and instar number in field and laboratory Manduca sexta. Proc R Soc B Biol Sci. 2007;274:977-81.

63. Nijhout HF. A THRESHOLD SIZE FOR METAMORPHOSIS IN THE TOBACCO HORNWORM, MANDUCA SEXTA (L.). Biol Bull. 1975;149:214-25.

64. Chafino S, Ureña E, Casanova J, Casacuberta E, Franch-Marro X, Martín D. Upregulation of E93 Gene Expression Acts as the Trigger for Metamorphosis Independently of the Threshold Size in the Beetle Tribolium castaneum. Cell Rep. 2019;27:1039-1049.e2. 65. Altstein M, Nässel DR. Neuropeptide Signaling in Insects. 2010;155-65. Available from: http://link.springer.com/10.1007/978-1-4419-6902-6_8

66. Callier V, Frederik Nijhout $\mathrm{H}$. Plasticity of insect body size in response to oxygen: 
Integrating molecular and physiological mechanisms. Curr Opin Insect Sci [Internet]. Elsevier Inc; 2014;1:59-65. Available from: http://dx.doi.org/10.1016/j.cois.2014.05.007

67. Dekanty A, Lavista-Llanos S, Irisarri M, Oldham S, Wappner P. The insulin-PI3K/TOR pathway induces a HIF-dependent transcriptional response in Drosophila by promoting nuclear localization of HIF- $\alpha$ /Sima. J Cell Sci. 2005;118:5431-41.

68. Gorr TA, Gassmann M, Wappner P. Sensing and responding to hypoxia via HIF in model invertebrates. J Insect Physiol. 2006;52:349-64.

69. Ratcliffe PJ. Oxygen sensing and hypoxia signalling pathways in animals: The implications of physiology for cancer. J Physiol. 2013;591:2027-42.

70. Katoh K, Standley DM. MAFFT multiple sequence alignment software version 7: Improvements in performance and usability. Mol Biol Evol. 2013;30:772-80.

71. Capella-Gutiérrez S, Silla-Martínez JM, Gabaldón T. trimAl: A tool for automated alignment trimming in large-scale phylogenetic analyses. Bioinformatics. 2009;25:1972-3. 72. Price MN, Dehal PS, Arkin AP. FastTree 2 - Approximately maximum-likelihood trees for large alignments. PLoS One. 2010;5.

73. Jones DT, Taylor WR, Thornton JM. The rapid generation of mutation data matrices from protein sequences. Bioinformatics. 1992;8:275-82.

74. Schinko J, Posnien N, Kittelmann S, Koniszewski N, Bucher G. Single and double wholemount in situ hybridization in red flour beetle (Tribolium) embryos. Cold Spring Harb Protoc. 2009;4:1-5.

75. Schindelin J, Arganda-Carreras I, Frise E, Kaynig V, Longair M, Pietzsch T, et al. Fiji: An open source platform for biological image analysis. Nat Methods. 2012;9:676-82.

\section{Figure legends}

Figure 1) Genomic evolution of subtilisin-like prohormone convertases. A) Gene tree (based on protein sequence) showing the clustering of prohormone convertases and furins in different animal groups, including most major insect taxa. SKI-1-poteins were used as outgroup. Node labels indicate local bootstrap support values. Full species names and protein sequences used in the alignment are given in the supplementary table S1. B) Table showing presence absence distribution of PC-orthologues in insects and other animal groups. Overall, the system is conserved. A PC1/3 but no PC2 orthologue is present in cnidarians, identifying PC1/3 as the evolutionary older convertase. Only dipterans and C. elegans have lost PC1/3. All insects have lost the PC7 genes, whereas it was retained in the myriapod Strigamia maritima and the lophotrochozoan Lottia gigantea. A group of basal PCs closely related to PC7 genes is present in the Cnidarian Nematostella. Lottia and Strigamia have, in addition to PC7 proteins, sequences that appear to be closely related to these pre-bilaterian basal PCs. The bilaterian invertebrate species each have furin 1 and 2 , whereas only 1 furin protein is 
present in the cnidarians. Vertebrate furins have diverged into furin, PC4, PC5 and PACE4. *The Orchesella cincta sequence (furin1-1_Orchesella, see tree in A) does not group with any of the subgroups. Based on the presence of a second furin1 gene in the other included collembolan Folsomia candida (furin1-2_Folsomia) we assume that the Orchesella sequence might be a derived furin 1 duplicate, even though this is not supported by the alignment). All cartoons credited to PhyloPic/free licence, hemipteran: K. Garcia/PhyloPic. C) Protein structure of Tribolium PC1/3 and PC2 showing the N-terminal Signal Peptide (SP), S8-pro domain, S8-peptidase domain, and the $\mathrm{P} /$ homo $\mathrm{B}$ regulatory domain. This structure is similar to the one of vertebrates. A specific sequence motif including -RRGDL- conserved in vertebrates and possibly involved in the sorting of the proteins to secretory vesicles [31] is present in Tribolium PC2 but bot PC1/3.

Figure 2) Divergent expression of PC1/3 and PC2 in Tribolium. A) NBT/B-ZIP colorimetric stain of embryonic PC2 expression, showing the anterior half of an embryo of stage NS14 (stages acccording to Biffar \& Stollewerk, 2014). The signal is located to the ventral neuroectoderm (arrowheads) and prospective brain area (arrow). B) Fluorescent in situ stain of an embryo at stage NS15. PC2 expressing cells (red) are associated with the developing central nervous system in the ventral neuroectoderm (arrowheads) and the prospective brain (arrow). Note that not all neurons are visible in this projection: the anterior brain commissures are already present but are located on a deeper level. The strong staining in the first abdominal segment (seen in A and $\mathbf{B}$, asterisks) represents unspecific signal from the pleuropods. C) Ubiquitous $P C 2$ expression in the anterior larval nervous system (shown is the brain and suboesophageal ganglion (SOG)). Some more intense staining is visible in the anterior medial brain (arrow). D) PC1/3 expression in the larval nervous system is restricted to individual cells of the dorsal side of the suboesophageal ganglion (arrows) and the posterior brain (arrowheads). Those cells are present bilaterally, but signal is obscured by unspecific signal of trachea on the left side (encircled areas).

Figure 3) Reduced viability following RNAi targeting PC1/3 and PC2. A) Survival curves over 41 days after pupal injections, showing percentage of living beetles at the given time. Control (dsRed-dsRNA) $n=30, P C 2$ and PC1/3 $n=100$. Note that all beetles had hatched on day 3 after injection and that deaths occurring before were likely caused by the injection process itself. 
B, C) Appearance of PC2- and PC1/3-dsRNA injected pupae found dead 5 days post injection (dpi) (lateral views). Abdomina are thin and dried out, wings are not fully elongated and not closed dorsally indicating incomplete metamorphosis. D, E) Appearance of PC2- and PC1/3dsRNA injected beetles found dead 15 days post injection (dpi) (D: lateral view, E: dorsal view). Wings are fully elongated but elytra were found fully (D) or half open (E). Both appearances were found in either knockdown. Abdomina were very flat and dry.

Figure 4) Reduced fertility in PC1/3- and PC2-RNAi beetles. A) Both enzymes are essential for female fertility. Shown are the numbers of eggs laid per female in 24 hours at the given time. Number of eggs/female/24h was $>10$ in dsRed-dsRNA control beetles on day 9 and 16, and $>8$ on day 23. In both knockdowns the number of eggs was reduced to less than 2 at all times. B) A reduction in reproduction is observed in treated males as well. Average number of eggs laid in 24 hours by 5 wildtype females that were crossed to 1 control-/RNAi-male, averaged over 30 males of each background. Error bars indicate standard deviations, T-test p-values significance levels: $* * *(P<0.001), *(P<0.05)$.

Figure 5) Ovarial phenotypes underlying female infertility in PC1/3- and PC2-RNAi-beetles. In this analysis we distinguish two phenotypes: 'small' (ovaries are evidently smaller and thinner) and 'held-egg' (eggs accumulate in the oviduct without being laid). A) Size comparison between a wildtype ovary and a 'small' ovary from a PC2-RNAi background. B) Ovary displaying 'held egg' phenotype: multiple eggs stuck in the oviducts lead to a disarranged appearance of the whole ovary. C) RNAi targeting either PC1/3 or PC2 lead to similar portions of 'small-ovary' and 'held egg' phenotypes. Given are the percentages of ovarial phenotypes observed in females 14-15 days post injection. D) Wildtype ovary (one half) with germarium (marked with ' $\mathrm{g}$ ') at the tips of the ovarioles and growing oocytes. White arrowheads point to oocytes that reached a mature size. Lateral oviduct (red asterisks) is widened and contains mature eggs. E) 'Small-ovary' phenotype from PC2 background: In this milder form of the phenotype the proportions of the ovarioles are maintained but the size of the most mature oocytes is reduced (white arrowheads) when compared to wildtype ovaries. Oviducts (red asterisks) do not contain mature eggs. F) More severe 'small-ovary' (from PC1/3 background). Ovarioles remain slender due to the lack of growth of the oocytes, which often display a gap to the follicle envelope. Some oocytes are seen disintegrating (white arrows) 
and oviducts (asterisks) do not contain mature eggs. Mild and severe 'small ovary' phenotypes were seen in both PC1/3 and PC2 knockdowns. G) Ovary of an untreated beetle starved for 10 days after eclosion, showing a similar 'small-ovary' morphology. Oviducts (red asterisks) do not contain mature eggs. H) Higher magnification of a mature oocyte from a mild 'smallovary' phenotype. Similar to wildtype, the egg is oval, and the cell membrane is lined by the follicle cell sheet without gaps. I) High magnification of an oocyte from a severe 'small-ovary' background: The oocyte is not oval and detached from the follicle sheet (red arrows). This indicates an insufficient distribution of nutrients to the oocyte by the follicle cells. J) An oocyte of a 'starved' background displays a similar gap between the egg membrane and the follicle sheet (red arrow).

Figure 6) PC1/3 and PC2 RNAi affect larval growth and survival. All larvae were injected at 12 days after egg lay ( $4^{\text {th }}$ larval instar) at a concentration of $1 \mu \mathrm{g} / \mu \mathrm{l}$ unless stated otherwise. Blue circles indicate successful metamorphosis to pupae, red crosses mark deaths. A) Growth curves of individual larvae injected with dsRed-dsRNA $(n=5)$. Larvae undergo constant growth until a weight of app. $2 \mathrm{mg}$ is reached. Larvae lose weight during the pre-pupal stage and pupate between day 21 and 23 of development. One larva with transiently reduced growth (L5) pupated later but at a similar weight. These results were similar to untreated wildtype larvae (see figure S2). B) PC1/3-RNAi larvae $(n=8)$ show strongly reduced growth and $50 \%$ die prematurely. The other half reach the pupal stage with strong delay (all surviving spent more than 35 days at the larval stage). C) PC2-RNAi larvae $(n=8)$ initially show a better growth than PC1/3 larvae but then all suffer severe weight loss and die as larvae. Lethality is $100 \%$. D) Double PC1/3- and PC2-RNAi ( $1 \mu \mathrm{g} / \mu \mathrm{l}$ each) $(n=8)$ leads to a combined phenotype with no weight gain and early death. Total injected dsRNA concentration was $2 \mu \mathrm{g} / \mu \mathrm{l}$, see figure S2. E) Average growth curves of each RNAi treatment based on data shown in A-D and including a dsRed control injected at $2 \mu \mathrm{g} / \mu \mathrm{l}$, matching the total dsRNA concentration of D (individual curves not shown). Error bars indicate standard deviations. Green circles indicate the time interval between two data points in which $>50 \%$ of larvae completed a moult. Dashed lines mark the interval in which all (surviving) larvae underwent a moult. Green circles marked with dsR1 and dsR2 on day 13 indicate that $>50 \%$ of dsRed $1 \mu \mathrm{g} / \mu \mathrm{l}$ - and $2 \mu \mathrm{g} / \mu \mathrm{l}$-injected larvae underwent a moult between injection and first data acquisition. Black asterisks indicate that a small proportion $(<50 \%)$ of the PC2-RNAi larvae $(1 / 8)$ and of the PC1/3-PC2-RNAi larvae 
(3/8) underwent a moult in the respective time intervals. PC1/3-RNAi larvae undergo supernumerary moults while showing severely reduced weight gain. Graph only includes development to day 42 , after which no more moults occurred. Individual data of PC1/3 larval growth and moult cycle is given in table $\mathrm{S4}$.

Figure 7) Additive amontillado-like locked-in phenotypes following larval RNAi against PC2, PC1/3 or both. $\left.A / A^{\prime}\right)$ Cuticle preparations reveal duplicated cuticular structures like the terminal cerci (green arrows in $\mathbf{A}$ ) and mandibles (red and blue arrows in $\mathbf{A}^{\prime}$ ). The second cuticle is also visible laterally on the head (black asterisks in $\mathbf{A}^{\prime}$ ) B) Table giving proportion of larvae in each knockdown that died from a 'locked-in' phenotype (PC1/3-RNAi n=38 (with 19 surviving), $P C 2-R N A i n=20, P C 1 / 3+P C 2-R N A i n=21)$. C) Table showing proportions of individuals that completed 1 or 2 moults successfully (also including individuals that survived to the pupal stage (PC1/3-RNAi only) and individuals that died but showed no locked-in phenotype. 


\section{figure 1}

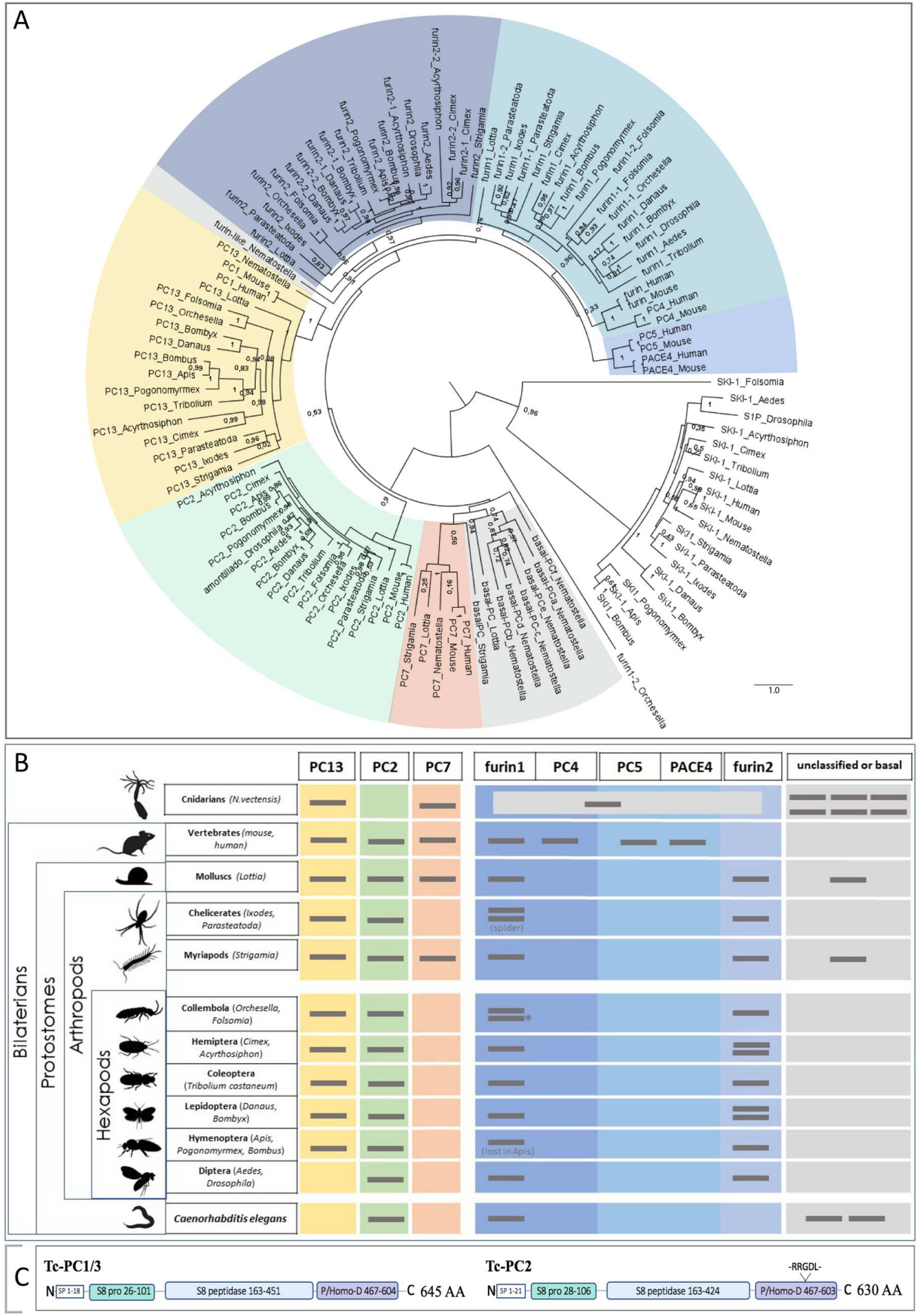


figure 2
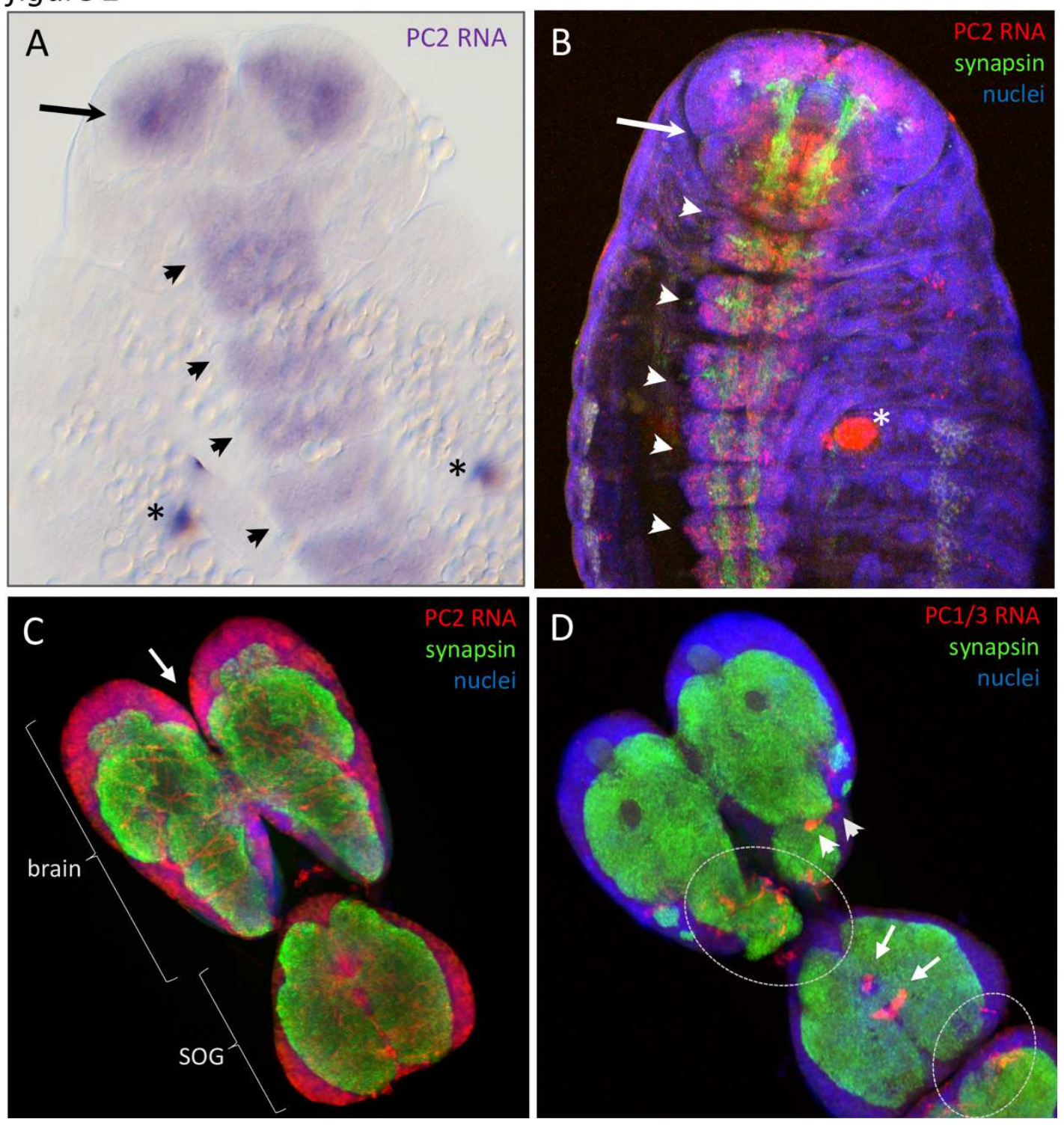


\section{figure 3}
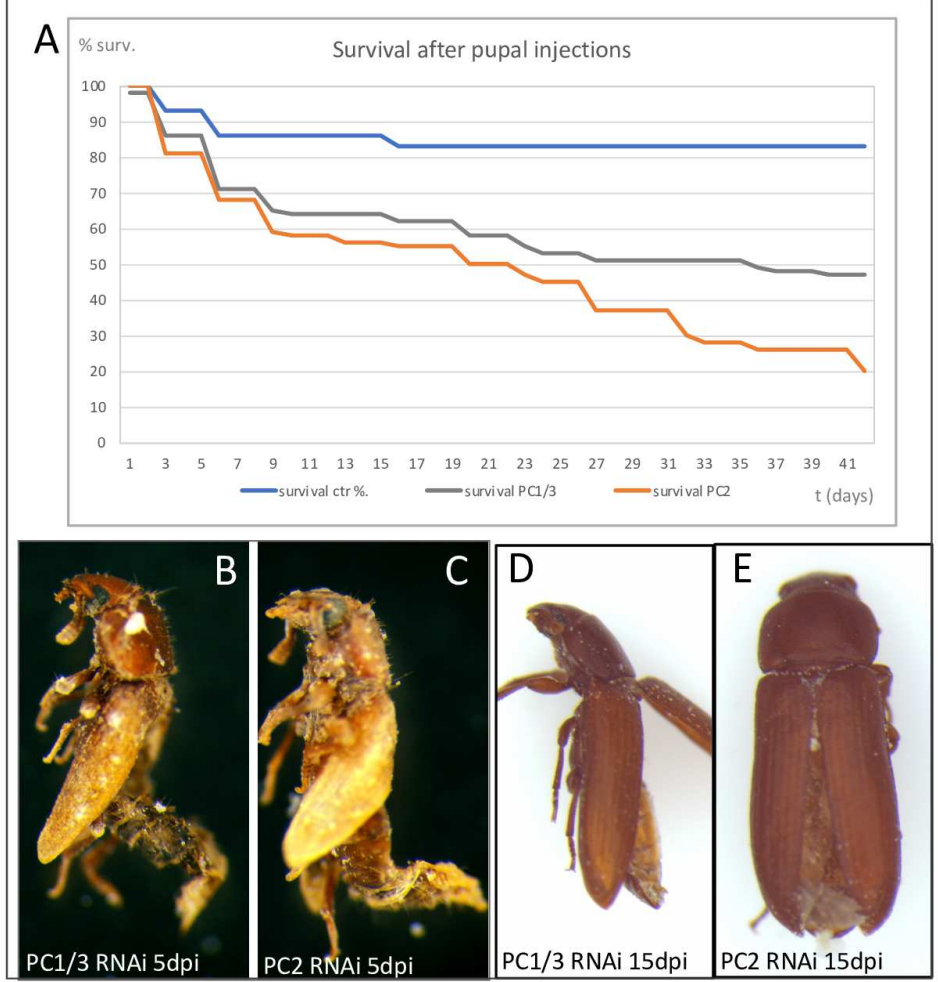

figure 4

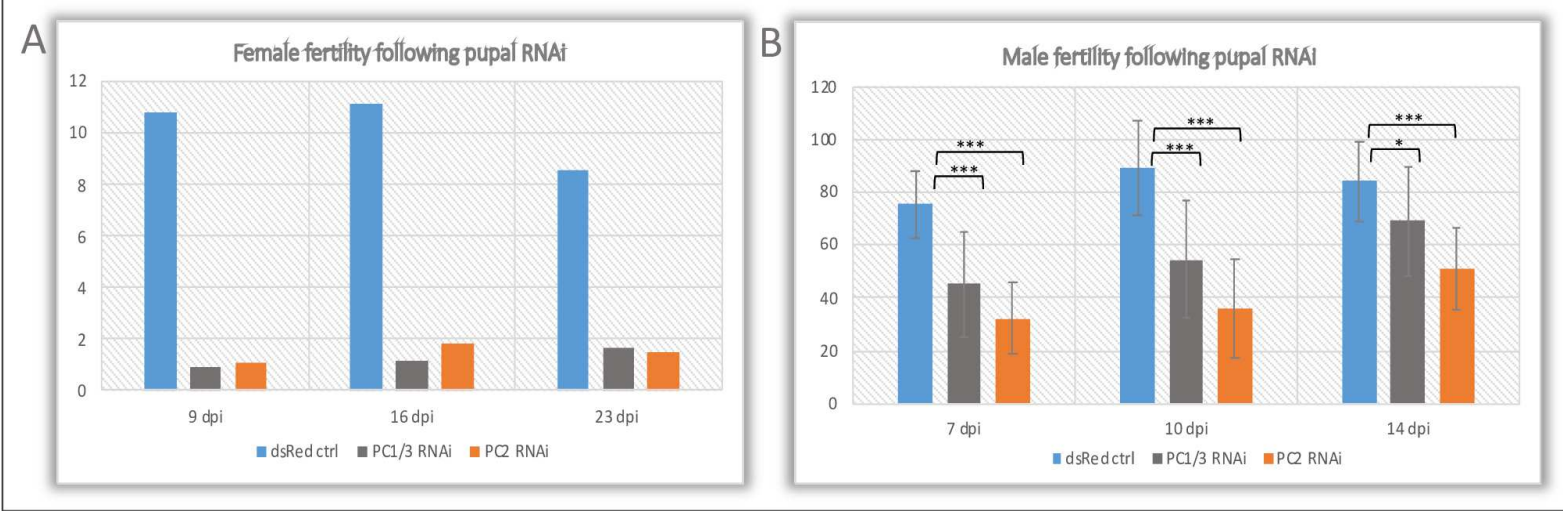


figure 5
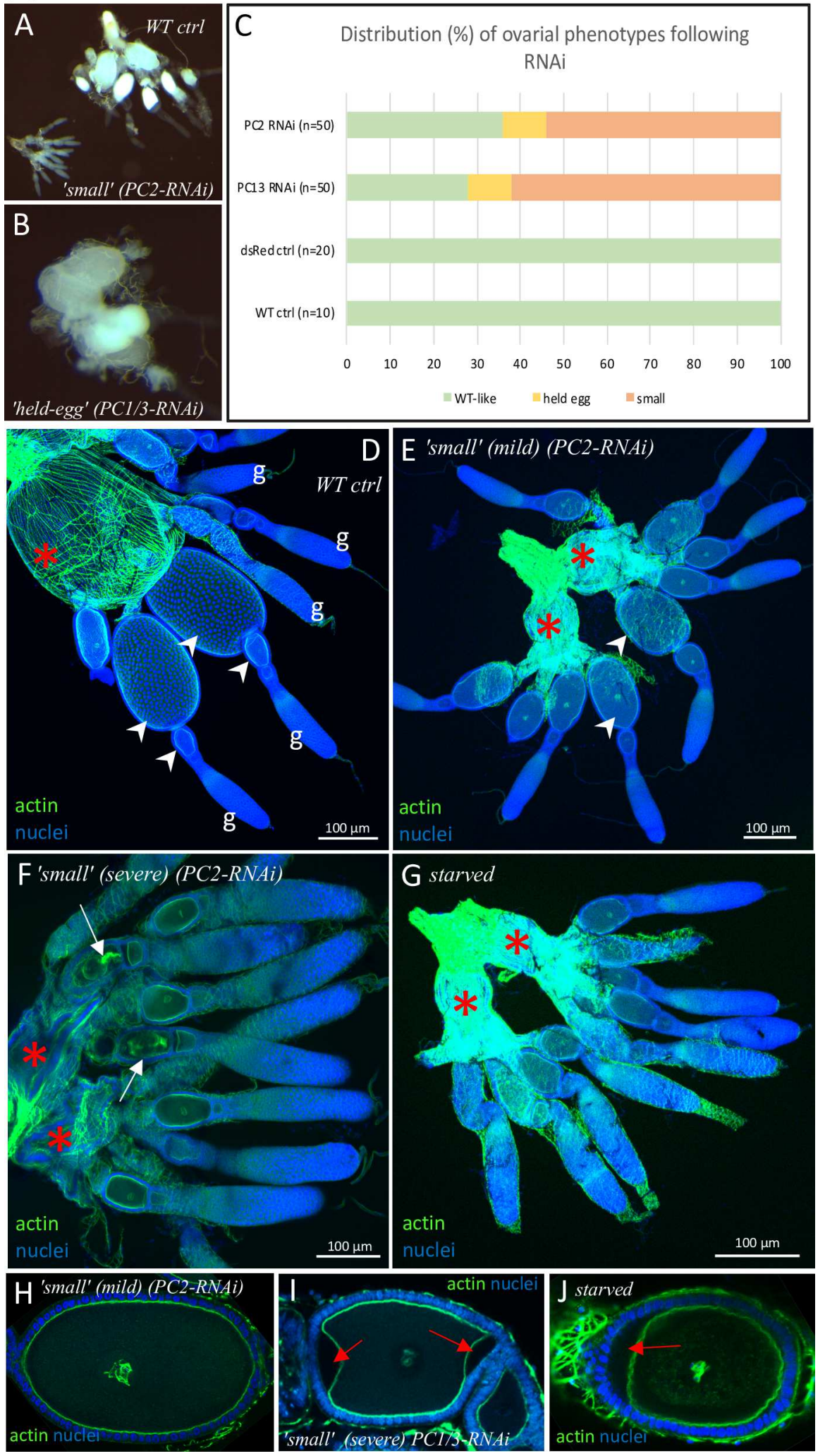
figure 6
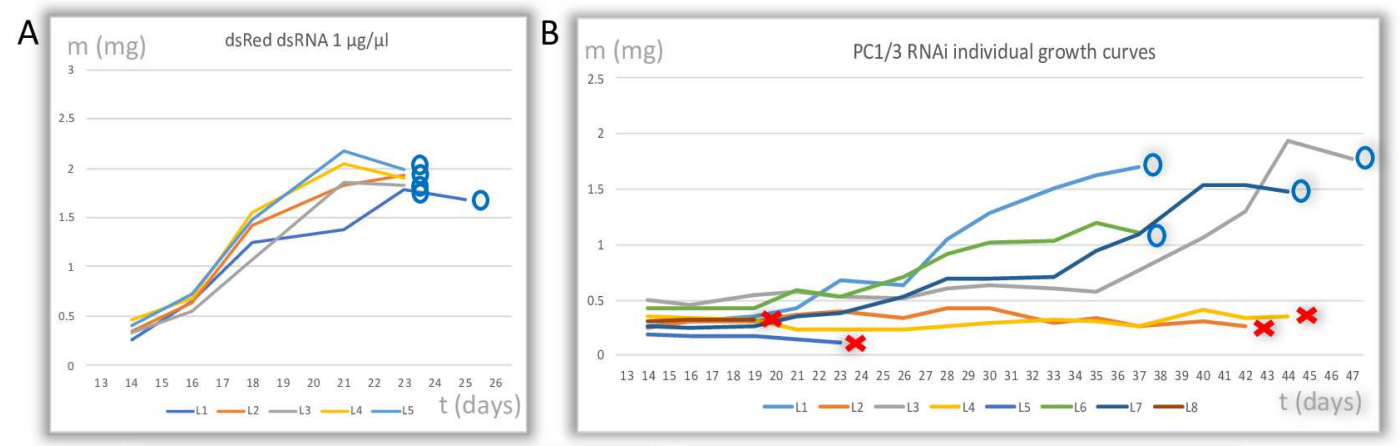

C $m(m g) \quad P C 2$ RNAi individual growth curves
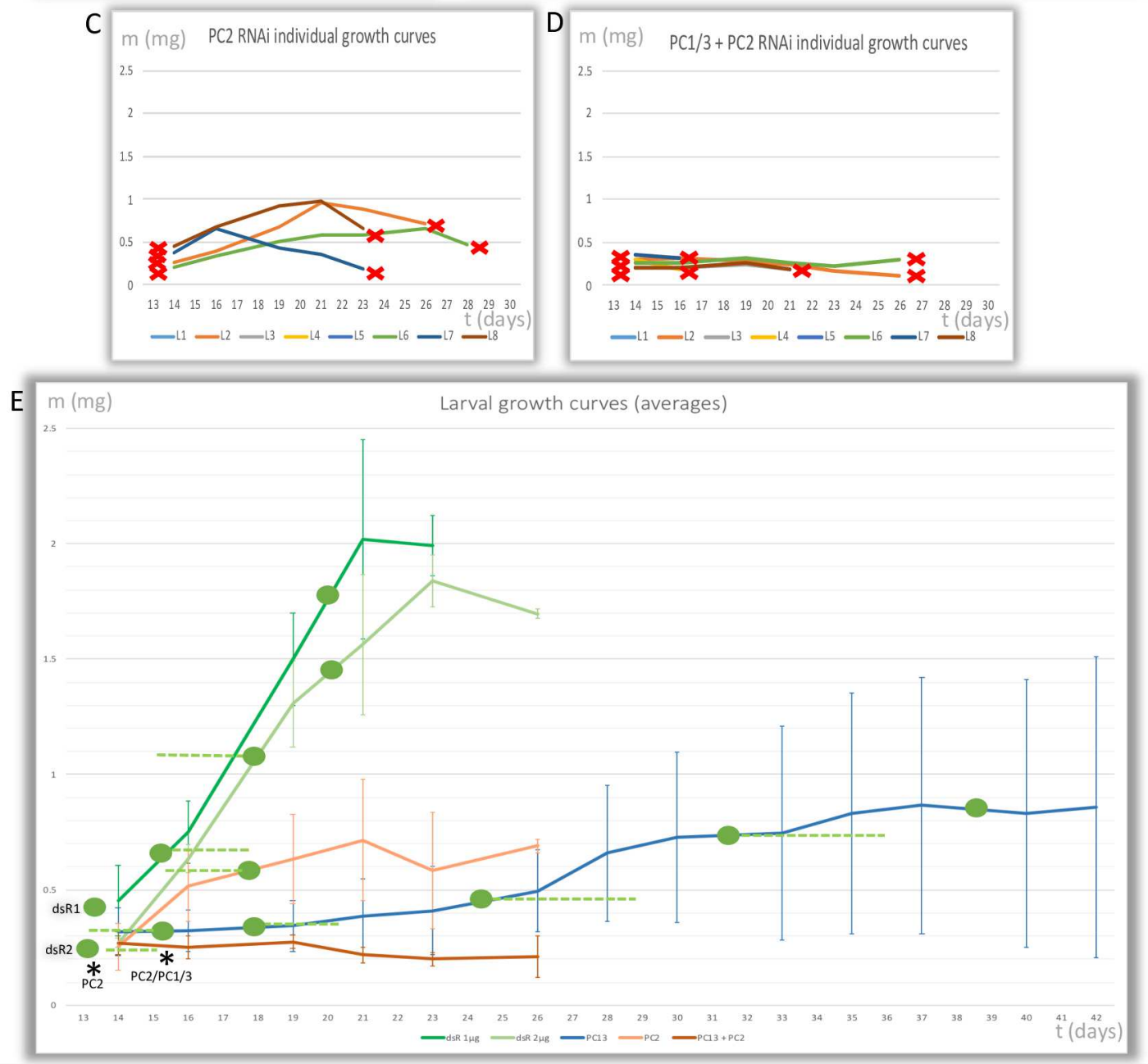
figure 7

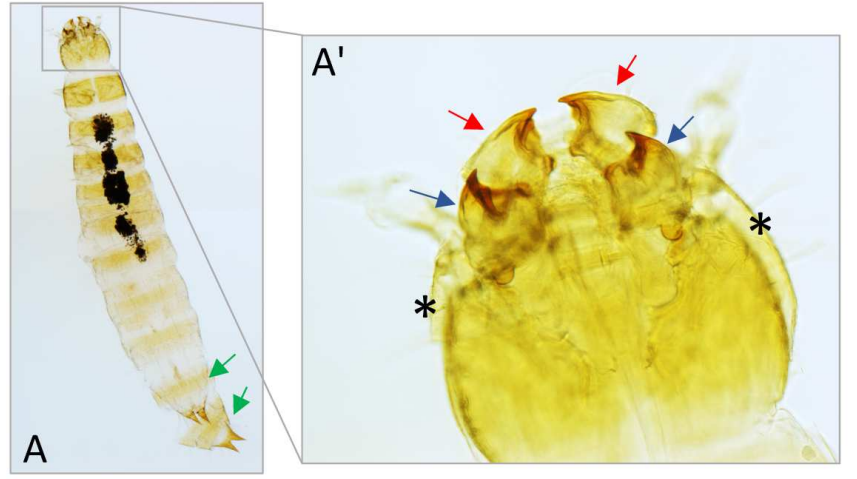

B

\begin{tabular}{lcccc}
\hline & ctrl & $\begin{array}{c}\text { PC1/3- } \\
\text { RNAi }\end{array}$ & $\begin{array}{c}\text { PC2- } \\
\text { RNAi }\end{array}$ & $\begin{array}{c}\text { PC1/3+ PC2- } \\
\text { RNAi }\end{array}$ \\
\hline $\begin{array}{l}\text { \% larvae dying from } \\
\text { locked-in phenotype }\end{array}$ & $0 \%$ & $13 \%$ & $75 \%$ & $100 \%$ \\
\hline C & & & & \\
\hline & ctrl & $\begin{array}{c}\text { PC1/3- } \\
\text { RNAi }\end{array}$ & $\begin{array}{c}\text { PC2- } \\
\text { RNAi }\end{array}$ & $\begin{array}{c}\text { PC1/3 + PC2- } \\
\text { RNAi }\end{array}$ \\
\hline $\begin{array}{c}1 \text { moult completed } \\
\text { after treatment }\end{array}$ & $100 \%$ & $100 \%$ & $65 \%$ & $33 \%$ \\
\hline $\begin{array}{c}\text { 2 moults completed } \\
\text { after treatment }\end{array}$ & $100 \%$ & $89 \%$ & $15 \%$ & $0 \%$ \\
\hline
\end{tabular}




\section{Figures}

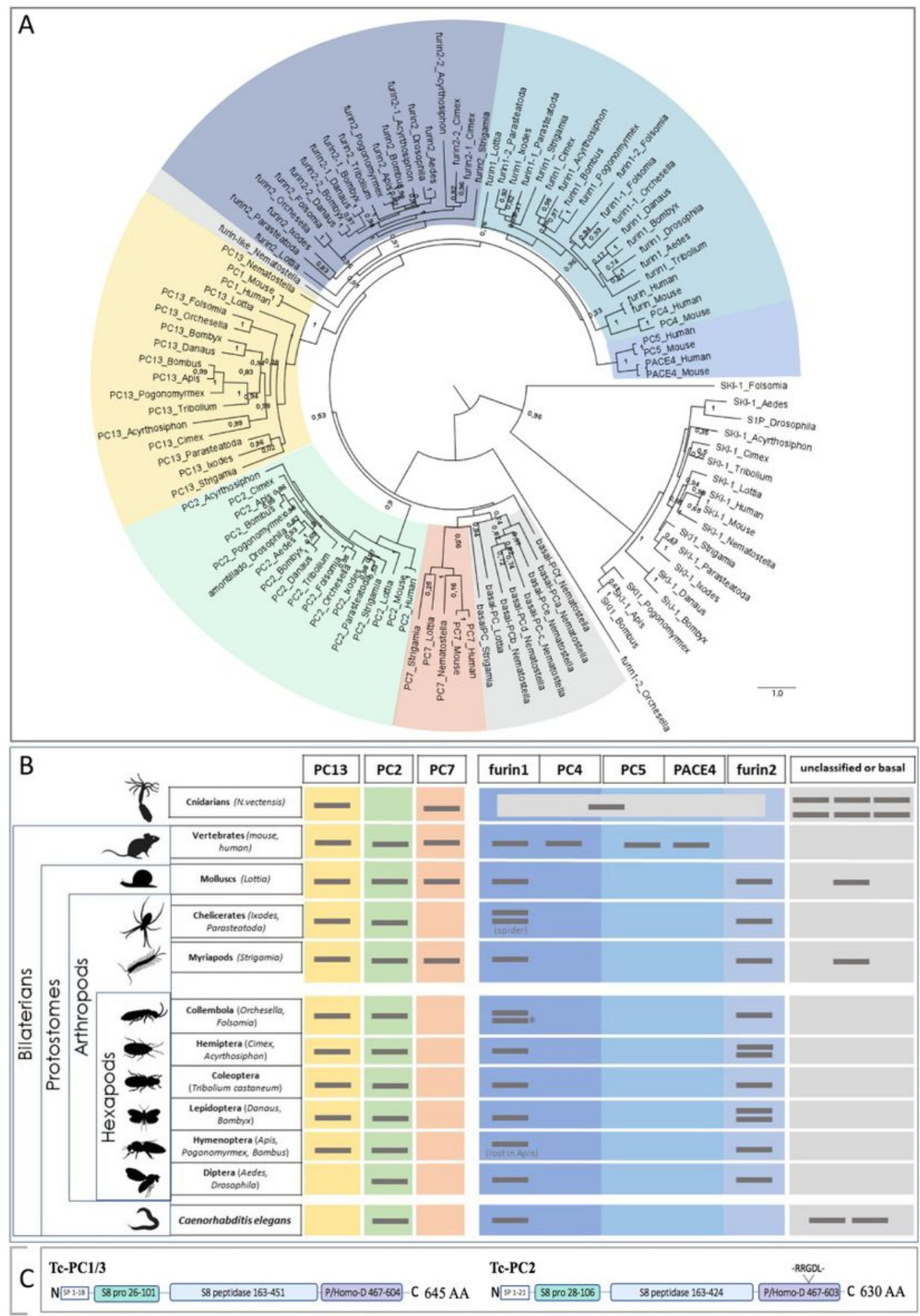

Figure 1

Genomic evolution of subtilisin-like prohormone convertases. A) Gene tree (based on protein sequence) showing the clustering of prohormone convertases and furins in different animal groups, including most major insect taxa. SKI-1-poteins were used as outgroup. Node labels indicate local bootstrap support 
values. Full species names and protein sequences used in the alignment are given in the supplementary table S1. B) Table showing presence absence distribution of PC-orthologues in insects and other animal groups. Overall, the system is conserved. A PC1/3 but no PC2 orthologue is present in cnidarians, identifying PC1/3 as the evolutionary older convertase. Only dipterans and C. elegans have lost PC1/3. All insects have lost the PC7 genes, whereas it was retained in the myriapod Strigamia maritima and the lophotrochozoan Lottia gigantea. A group of basal PCs closely related to PC7 genes is present in the Cnidarian Nematostella. Lottia and Strigamia have, in addition to PC7 proteins, sequences that appear to be closely related to these pre-bilaterian basal PCs. The bilaterian invertebrate species each have furin1 and 2 , whereas only 1 furin protein is present in the cnidarians. Vertebrate furins have diverged into furin, PC4, PC5 and PACE4. *The Orchesella cincta sequence (furin1-1_Orchesella, see tree in A) does not group with any of the subgroups. Based on the presence of a second furin 1 gene in the other included collembolan Folsomia candida (furin1-2_Folsomia) we assume that the Orchesella sequence might be a derived furin 1 duplicate, even though this is not supported by the alignment). All cartoons credited to PhyloPic/free licence, hemipteran: K. Garcia/PhyloPic. C) Protein structure of Tribolium PC1/3 and PC2 showing the N-terminal Signal Peptide (SP), S8-pro domain, S8-peptidase domain, and the P/homo B regulatory domain. This structure is similar to the one of vertebrates. A specific sequence motif including -RRGDL- conserved in vertebrates and possibly involved in the sorting of the proteins to secretory vesicles [31] is present in Tribolium PC2 but bot PC1/3. 

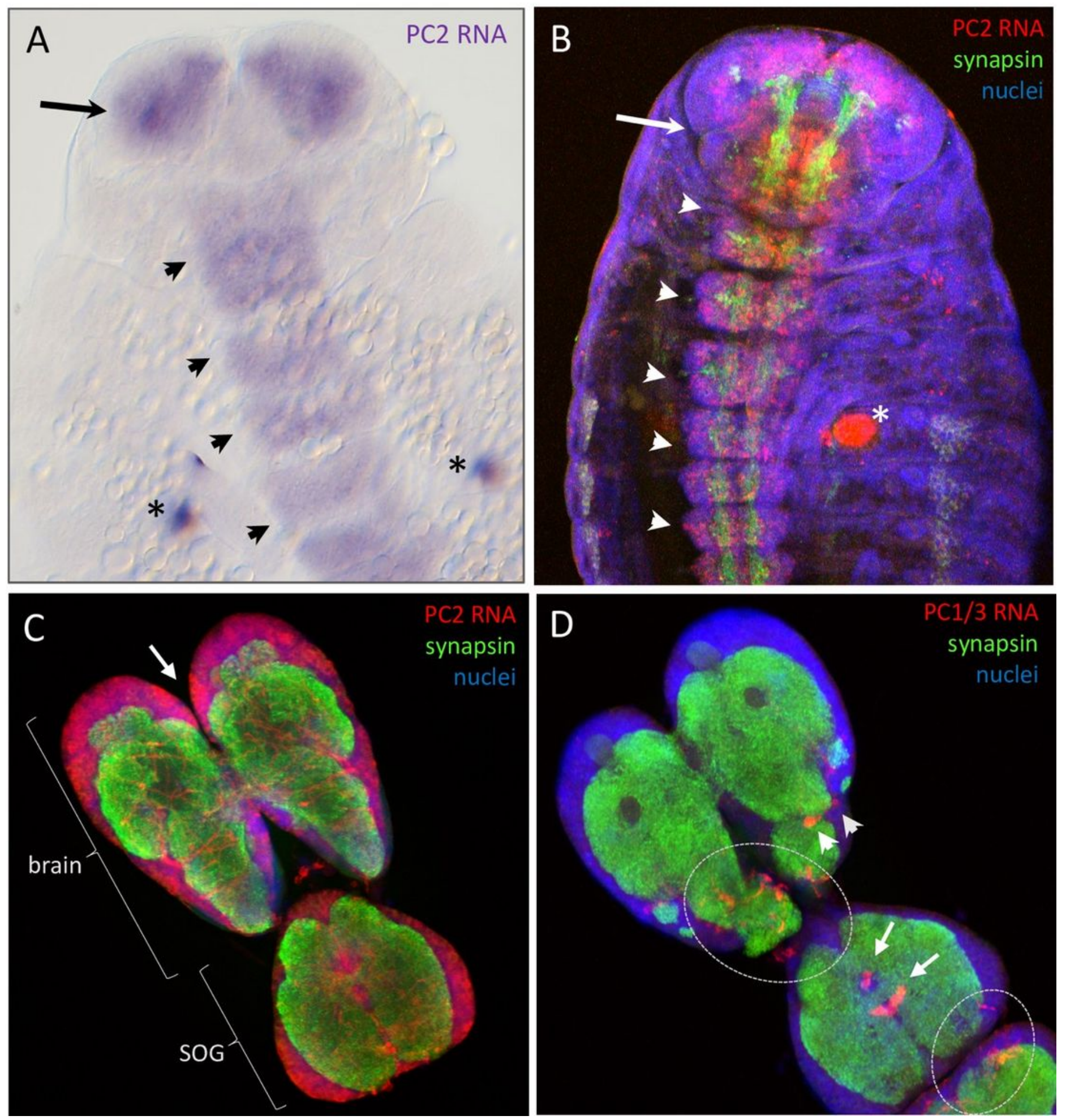

\section{Figure 2}

Divergent expression of PC1/3 and PC2 in Tribolium. A) NBT/B-ZIP colorimetric stain of embryonic PC2 expression, showing the anterior half of an embryo of stage NS14 (stages acccording to Biffar \& Stollewerk, 2014). The signal is located to the ventral neuroectoderm (arrowheads) and prospective brain area (arrow). B) Fluorescent in situ stain of an embryo at stage NS15. PC2 expressing cells (red) are associated with the developing central nervous system in the ventral neuroectoderm (arrowheads) and 
the prospective brain (arrow). Note that not all neurons are visible in this projection: the anterior brain commissures are already present but are located on a deeper level. The strong staining in the first abdominal segment (seen in $A$ and $B$, asterisks) represents unspecific signal from the pleuropods. C) Ubiquitous PC2 expression in the anterior larval nervous system (shown is the brain and suboesophageal ganglion (SOG)). Some more intense staining is visible in the anterior medial brain (arrow). D) PC1/3 expression in the larval nervous system is restricted to individual cells of the dorsal side of the suboesophageal ganglion (arrows) and the posterior brain (arrowheads). Those cells are present bilaterally, but signal is obscured by unspecific signal of trachea on the left side (encircled areas). 


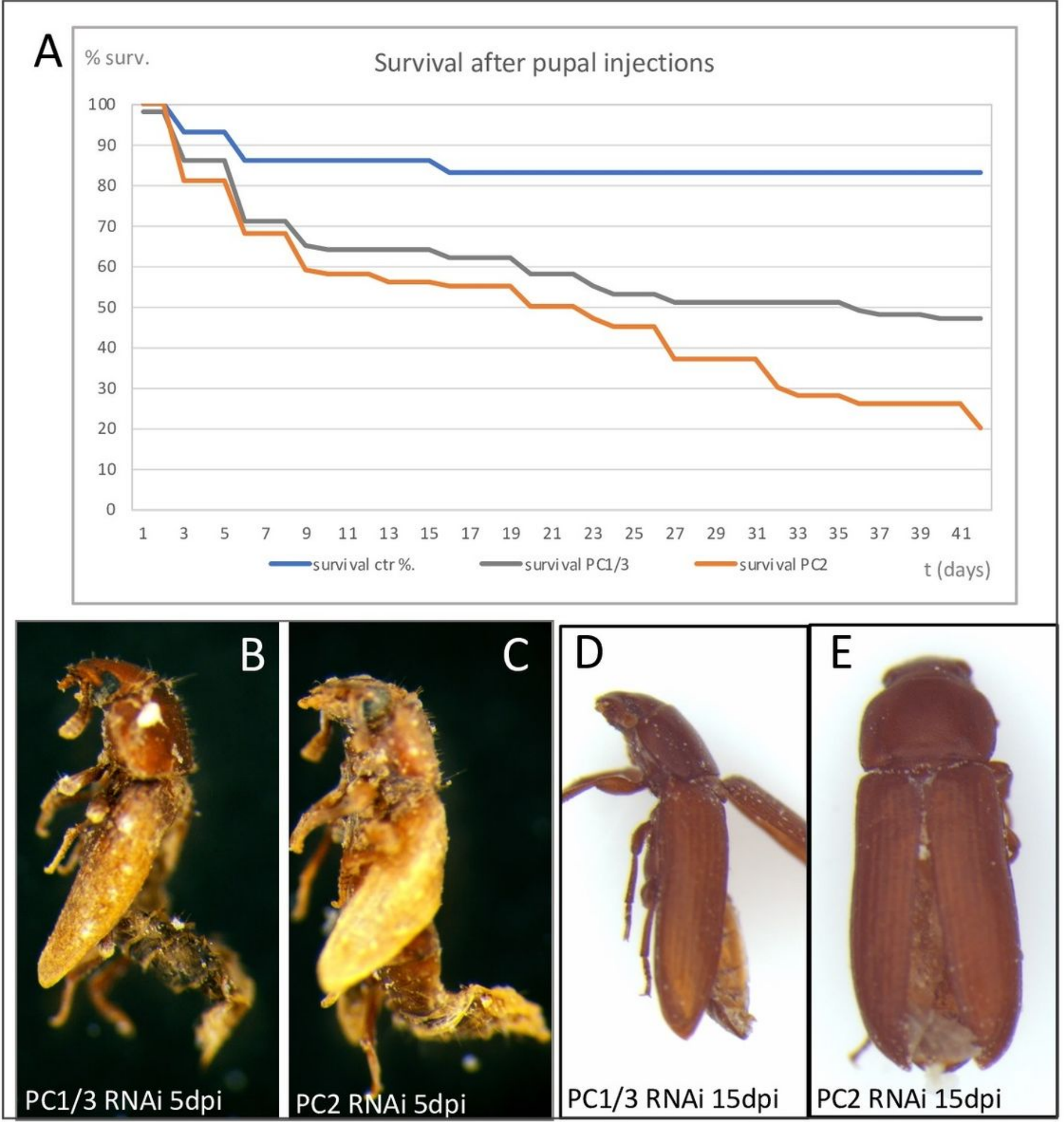

\section{Figure 3}

Reduced viability following RNAi targeting PC1/3 and PC2. A) Survival curves over 41 days after pupal injections, showing percentage of living beetles at the given time. Control (dsRed-dsRNA) $n=30, P C 2$ and $P C 1 / 3 n=100$. Note that all beetles had hatched on day 3 after injection and that deaths occurring before were likely caused by the injection process itself. B, C) Appearance of PC2- and PC1/3-dsRNA injected pupae found dead 5 days post injection (dpi) (lateral views). Abdomina are thin and dried out, wings are 
not fully elongated and not closed dorsally indicating incomplete metamorphosis. D, E) Appearance of PC2- and PC1/3- dsRNA injected beetles found dead 15 days post injection (dpi) (D: lateral view, E: dorsal view). Wings are fully elongated but elytra were found fully (D) or half open (E). Both appearances were found in either knockdown. Abdomina were very flat and dry.

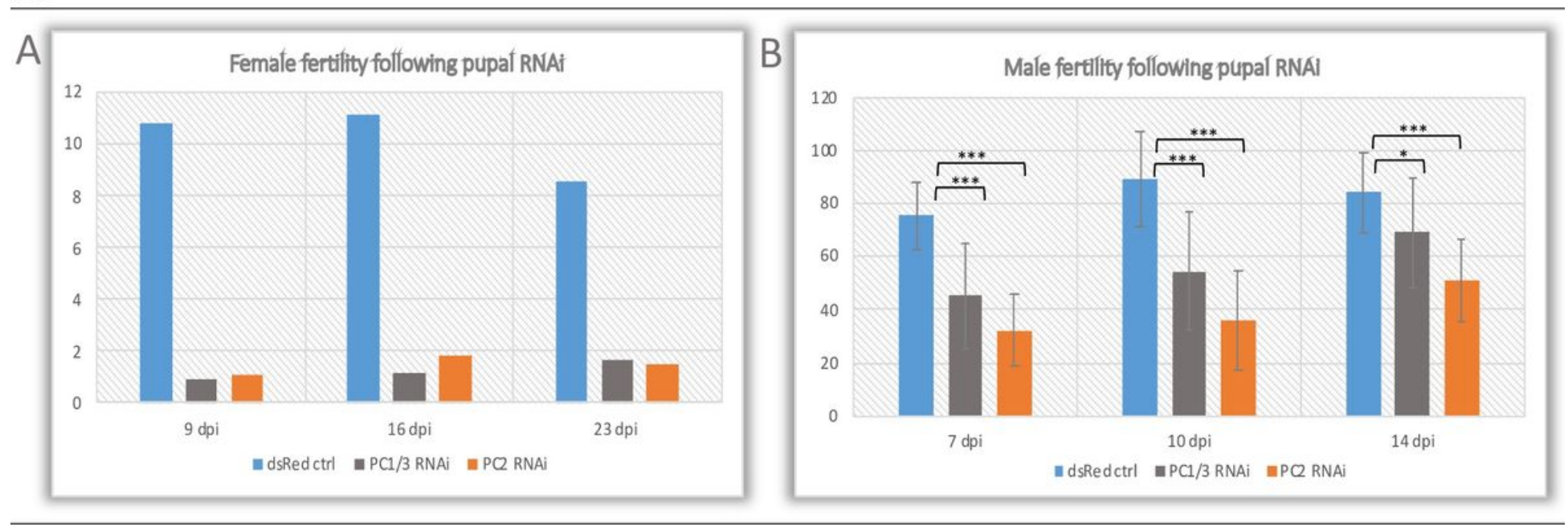

Figure 4

Reduced fertility in PC1/3- and PC2-RNAi beetles. A) Both enzymes are essential for female fertility. Shown are the numbers of eggs laid per female in 24 hours at the given time. Number of eggs/female/24h was $>10$ in dsRed-dsRNA control beetles on day 9 and 16 , and $>8$ on day 23 . In both knockdowns the number of eggs was reduced to less than 2 at all times. B) A reduction in reproduction is observed in treated males as well. Average number of eggs laid in 24 hours by 5 wildtype females that were crossed to 1 control-/RNAi-male, averaged over 30 males of each background. Error bars indicate standard deviations, T-test $p$-values significance levels: $\star \star \star ~(P<0.001), *(P<0.05)$. 

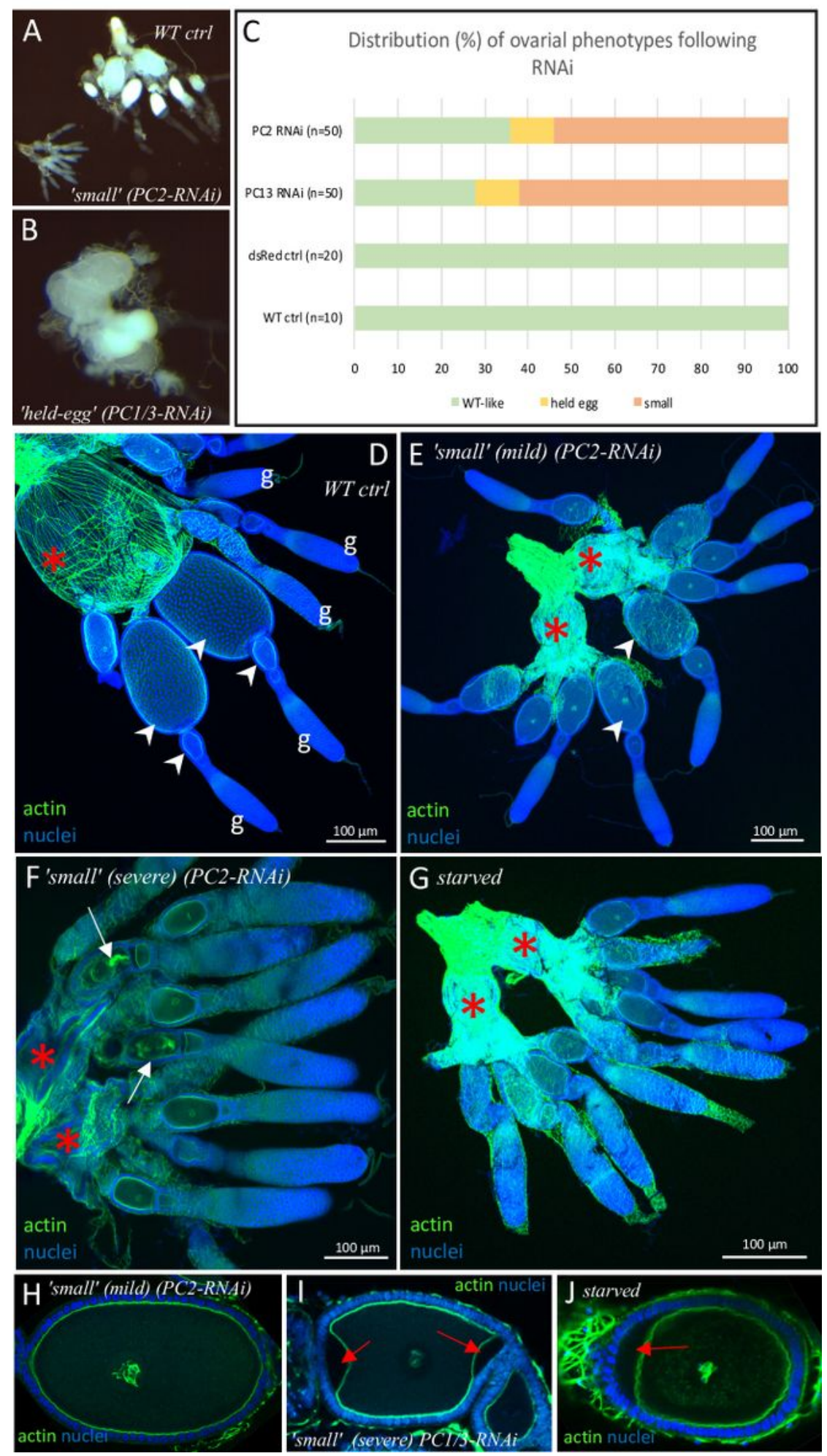

\section{Figure 5}

Ovarial phenotypes underlying female infertility in PC1/3- and PC2-RNAi-beetles. In this analysis we distinguish two phenotypes: 'small' (ovaries are evidently smaller and thinner) and 'held-egg' (eggs accumulate in the oviduct without being laid). A) Size comparison between a wildtype ovary and a 'small' ovary from a PC2-RNAi background. B) Ovary displaying 'held egg' phenotype: multiple eggs stuck in the oviducts lead to a disarranged appearance of the whole ovary. C) RNAi targeting either PC1/3 or PC2 lead 
to similar portions of 'small-ovary' and 'held egg' phenotypes. Given are the percentages of ovarial phenotypes observed in females 14-15 days post injection. D) Wildtype ovary (one half) with germarium (marked with ' $g$ ') at the tips of the ovarioles and growing oocytes. White arrowheads point to oocytes that reached a mature size. Lateral oviduct (red asterisks) is widened and contains mature eggs. E) 'Smallovary' phenotype from PC2 background: In this milder form of the phenotype the proportions of the ovarioles are maintained but the size of the most mature oocytes is reduced (white arrowheads) when compared to wildtype ovaries. Oviducts (red asterisks) do not contain mature eggs. F) More severe 'smallovary' (from PC1/3 background). Ovarioles remain slender due to the lack of growth of the oocytes, which often display a gap to the follicle envelope. Some oocytes are seen disintegrating (white arrows) and oviducts (asterisks) do not contain mature eggs. Mild and severe 'small ovary' phenotypes were seen in both PC1/3 and PC2 knockdowns. G) Ovary of an untreated beetle starved for 10 days after eclosion, showing a similar 'small-ovary' morphology. Oviducts (red asterisks) do not contain mature eggs. $\mathrm{H}$ ) Higher magnification of a mature oocyte from a mild 'smallovary' phenotype. Similar to wildtype, the egg is oval, and the cell membrane is lined by the follicle cell sheet without gaps. I) High magnification of an oocyte from a severe 'small-ovary' background: The oocyte is not oval and detached from the follicle sheet (red arrows). This indicates an insufficient distribution of nutrients to the oocyte by the follicle cells. J) An oocyte of a 'starved' background displays a similar gap between the egg membrane and the follicle sheet (red arrow). 


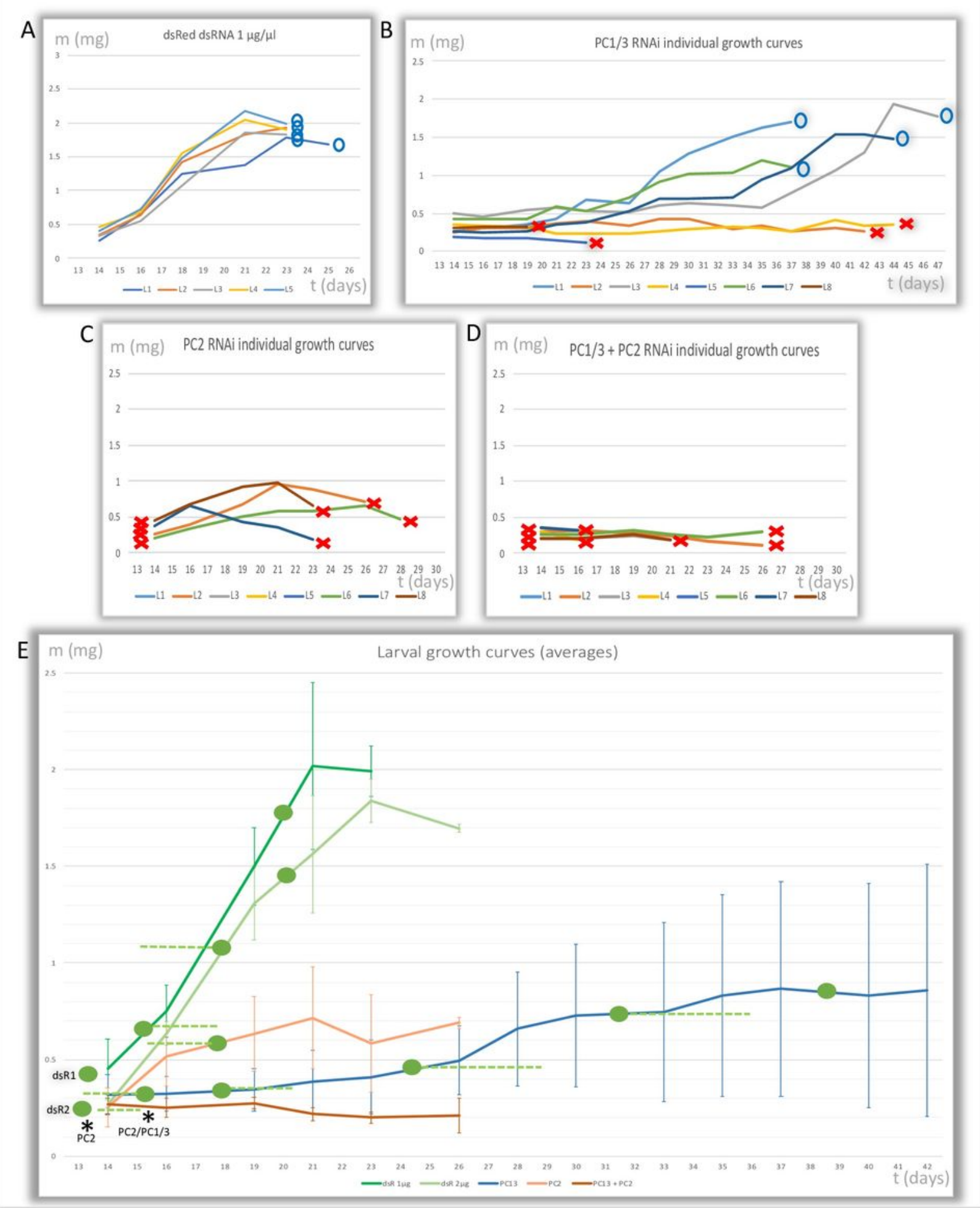

\section{Figure 6}

PC1/3 and PC2 RNAi affect larval growth and survival. All larvae were injected at 12 days after egg lay (4th larval instar) at a concentration of $1 \mu \mathrm{g} / \mu$ l unless stated otherwise. Blue circles indicate successful metamorphosis to pupae, red crosses mark deaths. A) Growth curves of individual larvae injected with dsRed-dsRNA ( $n=5)$. Larvae undergo constant growth until a weight of app. $2 \mathrm{mg}$ is reached. Larvae lose weight during the pre-pupal stage and pupate between day 21 and 23 of development. One larva with 
transiently reduced growth (L5) pupated later but at a similar weight. These results were similar to untreated wildtype larvae (see figure S2). B) PC1/3-RNAi larvae $(n=8)$ show strongly reduced growth and $50 \%$ die prematurely. The other half reach the pupal stage with strong delay (all surviving spent more than 35 days at the larval stage). C) PC2-RNAi larvae $(n=8)$ initially show a better growth than PC1/3 larvae but then all suffer severe weight loss and die as larvae. Lethality is $100 \%$. D) Double PC1/3- and PC2-RNAi $(1 \mu \mathrm{g} / \mu \mathrm{l}$ each) $(n=8)$ leads to a combined phenotype with no weight gain and early death. Total injected dsRNA concentration was $2 \mu \mathrm{g} / \mu \mathrm{l}$, see figure S2. E) Average growth curves of each RNAi treatment based on data shown in A-D and including a dsRed control injected at $2 \mu \mathrm{g} / \mu \mathrm{l}$, matching the total dsRNA concentration of D (individual curves not shown). Error bars indicate standard deviations. Green circles indicate the time interval between two data points in which $>50 \%$ of larvae completed a moult. Dashed lines mark the interval in which all (surviving) larvae underwent a moult. Green circles marked with dsR1 and dsR2 on day 13 indicate that $>50 \%$ of dsRed $1 \mu \mathrm{g} / \mu \mathrm{l}$ - and $2 \mu \mathrm{g} / \mu \mathrm{l}$-injected larvae underwent a moult between injection and first data acquisition. Black asterisks indicate that a small proportion ( $<50 \%$ ) of the PC2-RNAi larvae (1/8) and of the PC1/3-PC2-RNAi larvae (3/8) underwent a moult in the respective time intervals. PC1/3-RNAi larvae undergo supernumerary moults while showing severely reduced weight gain. Graph only includes development to day 42 , after which no more moults occurred. Individual data of PC1/3 larval growth and moult cycle is given in table S4.

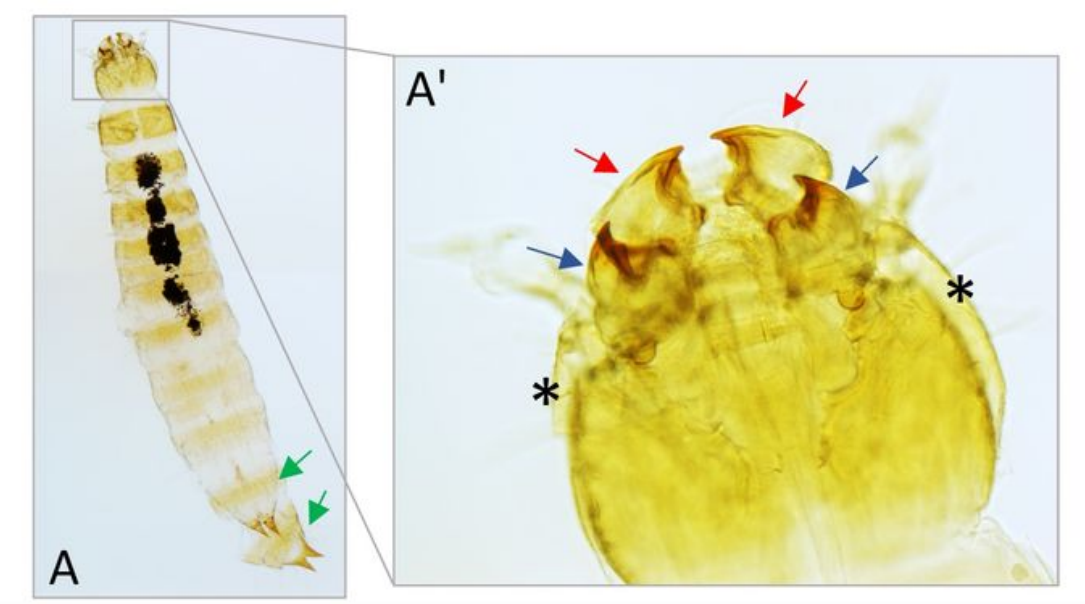

$\underline{B}$

\begin{tabular}{lcccc}
\hline & ctrl & $\begin{array}{c}\text { PC1/3- } \\
\text { RNAi }\end{array}$ & $\begin{array}{c}\text { PC2- } \\
\text { RNAi }\end{array}$ & $\begin{array}{c}\text { PC1/3 + PC2- } \\
\text { RNAi }\end{array}$ \\
\hline $\begin{array}{l}\text { \% larvae dying from } \\
\text { locked-in phenotype }\end{array}$ & $0 \%$ & $13 \%$ & $75 \%$ & $100 \%$ \\
\hline
\end{tabular}

C

\begin{tabular}{|c|c|c|c|c|}
\hline & ctrl & $\begin{array}{l}\text { PC1/3- } \\
\text { RNAi }\end{array}$ & $\begin{array}{l}\text { PC2- } \\
\text { RNAi }\end{array}$ & $\begin{array}{c}\mathrm{PC} 1 / 3+\mathrm{PC} 2- \\
\text { RNAi }\end{array}$ \\
\hline $\begin{array}{c}1 \text { moult completed } \\
\text { after treatment }\end{array}$ & $100 \%$ & $100 \%$ & $65 \%$ & $33 \%$ \\
\hline $\begin{array}{c}2 \text { moults completed } \\
\text { after treatment }\end{array}$ & $100 \%$ & $89 \%$ & $15 \%$ & $0 \%$ \\
\hline
\end{tabular}

\section{Figure 7}

Additive amontillado-like locked-in phenotypes following larval RNAi against PC2, PC1/3 or both. $A / A^{\prime}$ ) Cuticle preparations reveal duplicated cuticular structures like the terminal cerci (green arrows in A) and mandibles (red and blue arrows in $A^{\prime}$ ). The second cuticle is also visible laterally on the head (black asterisks in A') B) Table giving proportion of larvae in each knockdown that died from a 'locked-in' phenotype (PC1/3-RNAi n=38 (with 19 surviving), PC2-RNAi n=20, PC1/3+PC2-RNAi n=21). C) Table showing proportions of individuals that completed 1 or 2 moults successfully (also including individuals that survived to the pupal stage (PC1/3-RNAi only) and individuals that died but showed no locked-in phenotype. 


\section{Supplementary Files}

This is a list of supplementary files associated with this preprint. Click to download.

- SupplementarymaterialEvoDevo.pdf 